\title{
Shocks and quark-meson scatterings at large density
}

\author{
Eduardo Grossi $\odot,{ }^{1}$ Friederike J. Ihssen $\odot,{ }^{2}$ Jan M. Pawlowski, ${ }^{2,3}$ and Nicolas Wink ${ }^{2}$ \\ ${ }^{1}$ Center for Nuclear Theory, Department of Physics and Astronomy, Stony Brook University, Stony Brook, \\ New York 11794-3800, USA \\ ${ }^{2}$ Institut für Theoretische Physik, Universität Heidelberg, Philosophenweg 16, \\ 69120 Heidelberg, Germany \\ ${ }^{3}$ ExtreMe Matter Institute EMMI, GSI, Planckstrasse 1, 64291 Darmstadt, Germany
}

(Received 22 February 2021; accepted 29 June 2021; published 30 July 2021)

\begin{abstract}
We discuss the phase structure of the two-flavor quark-meson model including quantum, thermal, density and critical fluctuations with the functional renormalization group. This study combines two technical advances in the literature, that are also chiefly important for the quantitative access of the phase boundary of QCD at large density or baryon-chemical potential. Specifically we allow for the formation and propagation of shocks as well as a fully self-consistent computation of the order parameter potential for chiral symmetry breaking.
\end{abstract}

DOI: 10.1103/PhysRevD.104.016028

\section{INTRODUCTION}

The theoretical access to the QCD phase structure at large densities is chiefly important for our understanding of running and planned heavy-ion experiments; for reviews see [1-10]. At large densities, functional approaches, both within QCD and in low-energy effective models (LEFTs), have made rapid progress within the past two decades-for results in the present context with the functional renormalization group (FRG) see e.g., [11-19]; for results with Dyson-Schwinger equations (DSEs) see e.g., [8,9,20-24]. At small densities these studies are accompanied by respective lattice studies; see e.g., [25-32]. By now the results from both lattice and functional methods agree at small densities. In turn, at larger densities the lattice is hampered by the sign problem, while the approximations to the full QCD effective action within functional approaches require systematic qualitative improvements.

Chiefly important are the introduction of a Fierzcomplete basis of four-quark scattering vertices as well as quantitative access to order parameter potentials for homogeneous and inhomogeneous condensates. The latter allows us to discuss the eminently important question of the location of phase transition lines, that of the symmetrybreaking pattern and the order of the phase transitions. It has been shown in the past decade that functional QCD flows toward QCD-assisted low-energy effective models

Published by the American Physical Society under the terms of the Creative Commons Attribution 4.0 International license. Further distribution of this work must maintain attribution to the author(s) and the published article's title, journal citation, and DOI. Funded by SCOAP. for energy scales below $1 \mathrm{GeV}$; for a detailed discussion see in particular the recent works [12,17]. With dynamical hadronization [33-36] the LEFT is the (Polyakov-loopenhanced) quark-meson model (QM model), or more generally the quark-hadron model. For recent FRG works with the $(\mathrm{P}) \mathrm{QM}$ model on the phase structure of QCD relevant for the present work see e.g., [16,37-61]; for a recent overview see [17]. This emergence of LEFTs from first principle QCD flows is well understood and quantitatively explored in the vacuum; see [34,62-66]. It entails that the infrared critical dynamics is dominated by the lowenergy fluctuations of quarks and hadrons. For small baryon-chemical potentials, $\mu_{B} / T \lesssim 4$, the relevant hadronic degrees of freedom are simply the pseudoscalar pions and the sigma mode; see $[9,12,14,23,24]$. In turn, for baryon-chemical potentials $\mu_{B} \lesssim 4 / T$ the situation is less clear, but we expect sizable diquark contributions; see [14].

In the present work we make significant steps toward such a quantitative control of the phase structure of highdensity QCD within functional methods. It combines two systematic advances in the past years: The first one was the development of self-consistent approximations for the computation of order parameter potentials, [47]. The second one was the development of a numerical approach for solving flow equations that also enables us to discuss discontinuities in the flows such as shocks that are potentially relevant for the correct description of firstand second-order phase transitions [67]. Within this approach we compute the phase structure of the QM model at finite temperature and density. An important benchmark is already provided in the large- $N_{f}$ limit with an infinite number of flavors. It is argued that within an 't Hooft-type limit we can mimic the two-flavor QM model well (or any 
other flavor) and in particular reproduce well its nonuniversal properties such as the location of the phase boundary. Moreover, in this limit the numerical approach with discontinuous Galerkin setup in [67] is fully developed and we have a quantitative access to the shock development and propagation. The respective results are compare with the currently most advanced approximation (including shocks) to the self-consistent approximation including the order parameter potentials in [47] for the $N_{f}=2$-flavor quark-meson model. The results include also the regime $\mu_{B} / T \gtrsim 4$. In this regime the current model has to be augmented with a diquark channel which is done in a forthcoming work. Still, the present work is a necessary and important study also in this regime.

\section{QUARK-MESON MODEL}

The quark-meson model describes the dynamics of quarks and mesons at low energy. Within functional QCD this LEFT emerges naturally from the momentum scale flow of the theory at momentum or cutoff scales $k \lesssim 1 \mathrm{GeV}[12,34,62-66,68]$. In this regime the gluonic degrees of freedom decouple from the dynamics due to the gluonic mass gap in QCD-for a detailed discussion see $[12,17,65]$; for a discussion of the emergent LEFT see [66].

\section{A. Emergent LEFTs and their range of validity}

The key ingredient for this emergence is the scaledependent four-quark scattering, whose dynamics at large momentum scales is driven by a box diagram with a twogluon exchange between quark currents. For the discussion of its low-momentum behavior we restrict ourselves to the momentum-independent tensor structures, that is, ten tensor structures in two-flavor QCD and 28 (32) tensor structures in three-flavor QCD, the relevant cases for the discussion of the phase structure of QCD. It has been shown in $[64,65]$ that in the vacuum the scalar-pseudoscalar channel is dominating the dynamics by far, both above and below the chiral symmetry-breaking scale of $k \approx 500 \mathrm{MeV}$ : Switching of all other channels leads to negligible effects for most physical observables. Moreover, in [14] it has been shown for $N_{f}=2$-flavor QCD in the chiral limit that qualitatively this dominance persists up to large densities or chemical potentials, $\mu_{B} / T_{c}(0) \approx 6$, where $T_{c}\left(\mu_{B}\right)$ is the chiral crossover or phase transition temperature at a given baryon-chemical potential $\mu_{B}$. This highly interesting first dominance study in QCD is based on qualitative approximations, and a conservative error estimate leads us to $\mu_{B} / T_{c}(0) \lesssim 4-8$ for the (total) dominance regime of the scalar-pseudoscalar channel.

This supports the computations in [12], where the phase structure of 2- and 2+1-flavor QCD was computed within a one-channel approximation (scalar-pseudoscalar) to the Fierz-complete tensor structure for $\mu_{B} / T\left(\mu_{B}\right) \lesssim 6$ or $\mu_{B} / T\left(\mu_{B}\right) \lesssim 4$. Then, dynamical hadronization takes into account multiscattering events of the resonant channels (multiscatterings of pions and the scalar $\sigma$ mode) that are relevant for the critical dynamics in a regime with secondor first-order transitions. In summary we estimate the reliability regime of the present approximations in functional QCD (see also respective considerations in DSEs $[9,23,24])$ to be

$$
\mu_{B} / T\left(\mu_{B}\right) \lesssim 4
$$

The critical end points (CEPs) computed both within the most recent FRG computations, $\mu_{B} / T\left(\mu_{B}\right)=5.59$ from [12], and DSE, $\mu_{B} / T\left(\mu_{B}\right)=5.54$ from [23], for the physical case of 2+1-flavor QCD agree well, which sustains the respective reliability of these estimates. Still it is not within the regime of quantitative reliability of the current approximation.

Consequently, (1) entails that for a quantitatively sound prediction of the CEP the current approximation to the full first principle QCD flow has to be improved systematically in two directions for chemical potentials $\mu_{B} / T\left(\mu_{B}\right) \gtrsim 4$ : First we need to include at least the dominant tensor structure at large densities, the color super conductor or diquark channel. This extension will be considered elsewhere. Second the self-consistent computation of the order parameter potential setup in [7] is required. This is done in the present work within a recently developed numerical approach that also allows the inclusion of the formation and propagation of shocks [67].

\section{B. Quark-meson model with the functional renormalization group}

In this section we briefly recapitulate the FRG approach to the (Polyakov-enhanced) QM model. The inclusion of the dynamical mesons as low-energy effective degrees of freedom has to be seen as an efficient and convenient bookkeeping device for the respective resonant interaction channels. In particular, this substitutes the rather tedious inclusion of the resonant parts of the higher-order scattering processes of quarks. Still, if used on a quantitative level, even for large UV-cutoff scales its effective action does not reduce to a simple local classical action. For more details and in particular its quantitative properties as an emergent low-energy theory in QCD we refer to [12,63-65]. Validity checks, benchmarks and bounds in comparison to QCD have been provided in [66].

As discussed before, in the present work we restrict ourselves to a globally rather qualitative approximation to the effective action. Here, we are predominantly concerned with the quantitative access to the effective potential of the chiral order parameter. The systematic inclusion of the present quantitative setup within functional QCD flows is straightforward due to the modular nature of the FRG approach and will be considered elsewhere. 
The scales of the present LEFT are gauged by the pion decay constant in the chiral limit. We use $f_{\pi, \chi}=88 \mathrm{MeV}$ and measure all other scales with these units.

We choose the UV-cutoff scale of the QM model as $\Lambda=650 \mathrm{MeV}$. We consider this to be a good compromise between integrating out as many momentum fluctuations as possible and stretching the validity bound of the LEFT. The momentum fluctuations with momentum scales $k \leq \Lambda$ are included with the FRG. This approach has been used intensively in the past 25 years for the inclusion of lowenergy dynamics of the QM model. For the setup of the flow equation for the effective action and the derivation of the respective flow equations for (field-dependent) couplings we refer to the FRG reviews; see [17,69-72]. Applications relevant for the present work can be found in $[14,47,73]$, and the derivations and flows for the present approximation can be found in [47].

The effective action $\Gamma_{k}[q, \bar{q}, \phi]$ of the $N_{f}$-flavor QM model is used in the following approximation:

$$
\begin{aligned}
\Gamma_{k}[q, \bar{q}, \phi]= & \int_{x}\left\{\bar{q}\left(\gamma_{\mu} \partial_{\mu}-\gamma_{0} \mu_{q}\right) q+\frac{1}{2}\left(\partial_{\mu} \phi\right)^{2}\right. \\
& \left.+h_{k}(\rho) \bar{q}\left(\tau_{0} \sigma+\tau \pi\right) q+V_{k}(\rho)-c_{\sigma} \sigma\right\},
\end{aligned}
$$

with $\tau_{\mu}$ being related to the Pauli matrices, $\tau=1 / 2\left(\mathbb{1}, i \gamma_{5} \sigma\right)$, and the quark-meson coupling incorporates the $\mathrm{SU}(2) \cong$ $\mathrm{SO}(3)$ symmetry of the pseudoscalar subgroup. The $\mathrm{O}(4)-$ scalar field $\phi$ and the respective $\mathrm{O}(4)$-invariant $\rho$ are given by

$$
\phi=(\sigma, \vec{\pi})^{T}, \quad \rho=\frac{1}{2} \phi^{2}=\frac{1}{2}\left(\vec{\pi}^{2}+\sigma^{2}\right) .
$$

In (2) we have also $\int_{x}=\int_{0}^{1 / T} d x_{0} \int d^{3} x$ as an abbreviation for the finite temperature spatial integration.

We emphasize that the Yukawa coupling $h_{k}(\rho)$ is considered fully field dependent. It multiplies the $\mathrm{O}(4)$ invariant operator $\bar{q} \tau \phi q$; hence, it only depends on the $\mathrm{O}(4)$-invariant $\rho$. The field dependence of the Yukawa coupling takes into account higher-order pointlike scatterings of the resonant scalar-pseudoscalar channels with the quark-antiquark pair. The inclusion of these processes is necessary for a fully consistent zeroth-order derivative expansion and has been introduced in [47]. For further works in Yukawa models with field-dependent Yukawa coupling see [57,74-77]. This is easily seen by performing a perturbative one-loop computation within the QM model. Then, the quark loop with $h(\rho)$ contributes to the full effective potential. Of course higher terms in the derivative expansion also contribute to the effective potential, but the Yukawa term contains no derivatives. Accordingly, its full field dependence should be accounted for in a consistent lowest-order derivative expansion.

Finally, the scalar effective potential $V_{k}(\rho)$ takes into account the remaining part of the higher-order scatterings of the mesons. The linear term introduces explicit chiral symmetry breaking (finite current quark masses). Evidently, it drops out on the right-hand side of the flow equation and does not run. Consequently, the full flow and hence the full effective potential $V_{k}$ do not know anything about explicit chiral symmetry breaking, and we do not consider it any further.

The next systematic step beyond the zeroth-order derivative expansion would be the inclusion of wave-function renormalizations $Z_{q}(\phi)$ and $Z_{\phi}(\phi)$ for quarks and mesons. This can be done either fully field dependent (first-order derivative expansion) or field independent (usually called $\mathrm{LPA}^{\prime}$ ). The latter approximation has been used in [47] together with a field-dependent Yukawa coupling. While technically in reach, we have chosen to drop these terms for the sake of concentrating on the quantitative discussion of the full effective potential. Hence, with (2) we assume implicitly

$$
Z_{q, k}(\rho)=1=Z_{\phi, k}(\rho) .
$$

The flow equation for the complete set of couplings, $h_{k}(\rho), V_{k}(\rho)$, and wave-function renormalizations can be found in [47]. We use the same setup here, including the choice of regulators, three-dimensional flat or Litim regulators [78].

For the effective potential we simply evaluate the flow for $\Gamma_{k}[q, \bar{q}, \phi]$ for constant scalar fields $\phi$ and vanishing quark fields $q, \bar{q}=0$. This leads us to

$$
\begin{aligned}
\partial_{t} V_{k}(\rho)= & \frac{k^{5}}{12 \pi}\left[-\frac{4 N_{f} N_{c}}{\epsilon_{k}^{q}}\left(1-n_{f}\left(\epsilon_{k}^{q}+\mu\right)-n_{f}\left(\epsilon_{k}^{q}-\mu\right)\right)\right. \\
& \left.+\frac{N_{f}^{2}-1}{\epsilon_{k}^{\pi}}\left(1+2 n_{B}\left(\epsilon_{k}^{\pi}\right)\right)+\frac{1}{\epsilon_{k}^{\sigma}}\left(1+2 n_{B}\left(\epsilon_{k}^{\sigma}\right)\right)\right],
\end{aligned}
$$

with $\rho$-dependent quark- and meson-dispersion relations

$\epsilon_{k}^{\phi}(\rho)=\sqrt{k^{2}+m_{\phi, k}^{2}(\rho)}, \quad \epsilon_{k}^{q}(\rho)=\sqrt{k^{2}+m_{q, k}^{2}(\rho)}$,

and the RG time $t=\ln k / \Lambda$. The $\mathrm{RG}$ time involves a reference scale in the logarithm, which we have set to be the initial scale. The masses $m_{q}$ and $m_{\phi}$ are obtained by evaluating the respective two-point functions at constant fields. Note that $m_{q}$ and $m_{\phi}$ are the curvature and not the pole or screening masses of quarks and mesons; for respective definitions and discussions see [46].

The meson curvature masses are defined with

$$
\begin{aligned}
& m_{\pi, k}^{2}(\rho)=\partial_{\rho} V_{k}(\rho), \\
& m_{\sigma, k}^{2}(\rho)=\partial_{\rho} V_{k}(\rho)+2 \rho \partial_{\rho}^{2} V_{k}(\rho)
\end{aligned}
$$

and hence are curvature coefficients of the effective potentials. In turn, the quark mass is proportional to the Yukawa coupling: 


$$
m_{q, k}^{2}(\rho)=2 h_{k}(\rho)^{2} \rho .
$$

It is left to discuss the flow equation for the field-dependent Yukawa coupling; for details we again refer to [47]. We can project the flow for $\Gamma_{k}$ onto the Yukawa coupling $h(\rho)$ by evaluating the quark two-point function at vanishing quark and pion fields $q, \bar{q}, \boldsymbol{\pi}=0$ and constant $\sigma$. With (2) we arrive at

$$
\begin{aligned}
\Gamma_{q \bar{q}, k}^{(2)}[\sigma](p) \delta_{p, p^{\prime}} & =\left.\frac{\delta^{2} \Gamma[q, \bar{q}, \phi]}{\delta q(p) \delta \bar{q}\left(p^{\prime}\right)}\right|_{q, \bar{q}, \boldsymbol{\pi}=0} \\
& \simeq i \not p-\gamma_{0} \mu+h_{k}(\rho) \sigma-c_{\sigma} \sigma,
\end{aligned}
$$

where we have dropped the momentum conservation $\delta_{p, p^{\prime}}$ in the last line with $\delta_{p, p^{\prime}}=(2 \pi)^{4} \delta\left(p-p^{\prime}\right)$ in the vacuum.

Equation (9) reflects the fact that the Yukawa term simply is the $\rho$-dependent mass quark term $m_{q, k}(\sigma)=h_{k}(\rho) \sigma / 2$. Accordingly, the flow of the Yukawa coupling $h_{k}(\rho)$ can be derived from that of the scalar part of the quark two-point function: It is simply $\sigma / 2 \partial_{t} h_{k}(\rho)$ as $\partial_{t} c_{\sigma}=0$ by definition. Thus we get

$$
\partial_{t} h_{k}(\rho)=-\frac{1}{4 N_{c} N_{f}} \frac{1}{\sigma} \operatorname{Re} \operatorname{Tr} \Gamma_{q \bar{q}, k}^{(2)}[\sigma](p=0) .
$$

In (10) we have used that $\rho=\sigma^{2} / 2$ for $\pi=0$. The flow (10) is depicted in Fig. 1. From (10) and the approximation (2) we finally get

$$
\begin{aligned}
\partial_{t} h_{k}(\rho)= & 4 v_{3} h_{k}^{3}(\rho)\left[L_{(1,1)}^{(4)}\left(m_{q, k}^{2}, m_{\sigma, k}^{2} ; T, \mu\right)\right. \\
& \left.-\left(N_{f}^{2}-1\right) L_{(1,1)}^{(4)}\left(m_{q, k}^{2}, m_{\pi, k}^{2} ; T, \mu\right)\right] \\
& +16 v_{3} h_{k}(\rho) h_{k}^{\prime}(\rho) \rho\left[h_{k}(\rho)+\rho h_{k}^{\prime}(\rho)\right] \\
& \times L_{(1,1)}^{(4)}\left(m_{q, k}^{2}, m_{\sigma, k}^{2} ; T, \mu\right) \\
& -2 v_{3} k^{2}\left[\left(3 h_{k}^{\prime}(\rho)+2 \rho h_{k}^{\prime \prime}(\rho)\right) l_{1}^{(B, 4)}\left(m_{\sigma, k}^{2} ; T\right)\right. \\
& \left.+\left(N_{f}^{2}-1\right) h_{k}^{\prime}(\rho) l_{1}^{(B, 4)}\left(m_{\pi, k}^{2} ; T\right)\right]
\end{aligned}
$$

with

$$
\begin{aligned}
& v_{d-1}=\frac{1}{2^{d+1} \pi^{\frac{d}{2}} \Gamma\left(\frac{d}{2}\right)} . \\
& \partial_{t} h_{k}=-\frac{i}{4 N_{c} N_{f}}\left(-3-+\infty-\frac{1}{2}+\right)
\end{aligned}
$$

FIG. 1. Diagrammatic representation of the flow of the Yukawa coupling. The circled cross $\otimes$ depicts the regulator insertion $\partial_{t} R_{k}\left(\vec{p}^{2}\right)$ and the gray dots denote full vertices. The double lines depict the mesons, whereas the single line indicates the quark content. The arrows depict the quark number flow.
The threshold functions $l_{1}^{(B, 4)}$ originate in the bosonic loops in four dimensions. The $L_{(1,1)}^{(4)}$ originate in the mixed fermionic and bosonic contributions, again in four dimensions. Both functions are defined in Appendix H. The first four lines from (11) are contributions of the first two diagrams with mixed fermionic and bosonic loops in Fig. 1, whereas the fifth and the sixth lines correspond to the bosonic loop with the four-vertex.

\section{The large- $N$ limit}

Most of the numerical results in the present work are obtained in the large- $N$ limit of these equations, as it simplifies the numerical treatment significantly: It eliminates the $\sigma$ loop in the flow equation and hence the second derivative terms in the sigma meson mass term. We are left with only the pion loops as well as the quark loop. The pion loops constitute the flow of a pure $O(N)$ theory in the large$N$ limit as considered in [67] with discontinuous Galerkin methods. Such a nonlinear first-order system is solved using approximate Riemann solvers. These solvers rely on the assumption that the solution is dominated by one strong wave; for more details see Appendix A. This assumption holds if the flow is dominated by contributions of the pion and quark loops, which is always ensured in the large- $N$ limit.

This simplification is also helpful when considering systems of multiple differential equations. However, it will also be instructive to make a comparison between both the finite- $N_{f}$ case and the large- $N_{f}$ limit in the case with constant Yukawa coupling. Moreover, we can simulate the physics case, $N_{f}=2$ and $N_{c}=3$, with a suitable chosen large- $N$ limit.

To begin with we keep the ratio of color and flavor fixed to that of the $N_{f}=2$ quark-meson model in QCD:

$$
\frac{N_{c}}{N_{f}}=\frac{3}{2} .
$$

With (13a) we keep the flavor-color balance of QCD intact. This ensures that the contributions of the quark loop are not suppressed by $1 / N_{f}$. Moreover, the flavor-color ratio is certainly of crucial importance for e.g., the question of the existence and size of a quarkyonic phase. Finally, we consider the following generic rescaling of $\rho, V_{k}(\rho)$ and $h_{k}(\rho)$ :

$$
\begin{aligned}
\rho & \rightarrow \frac{N_{f}^{2}-1}{N_{\pi}} \rho, \\
V_{k}(\rho) & \rightarrow \frac{N_{f}^{2}-1}{N_{\pi}} V_{k}(\rho), \\
h_{k}(\rho) & \rightarrow \sqrt{\frac{N_{\pi}}{N_{f}^{2}-1}} h_{k}(\rho) .
\end{aligned}
$$


The factor $N_{\pi}$ in (13b) is introduced to simulate the flows of a quark-meson model with $N_{\pi}$ pions instead of one sigma meson and three pions. Both cases are relevant for the physics of two-flavor QCD or the two-flavor QM model.

In the chirally symmetric phase for large temperatures and cutoff scales, the pions and the sigma are degenerate on shell at $\rho=0$. The second derivative term vanishes $\left.2 \rho V^{\prime \prime}(\rho)\right|_{\rho=0}=0$, the on-shell $\sigma$ propagator agrees with the pion one, and the (on-shell) flow equation resembles that with four pions.

In turn, in the broken phase, the $\sigma$ mode develops a mass and quickly decouples from the dynamics of the system. Then, the (on-shell) dynamics of the theory is driven by the three (massless or light) pions. From previous FRG investigations of the quark-meson model as well as QCD we know that the mesonic dynamics in the broken phase is of subdominant importance for not too large chemical potential. This suggests that the $N=4$ case should mimic the two-flavor case best. A full discussion of the comparison is provided in Secs. IV B 1 and IV B 2.

With the limit $N_{f} \rightarrow \infty$ and (13) we derive the flow equations for large- $N_{f}$ Yukawa coupling, $h_{k}^{\mathrm{NN}}(\rho)$, and effective potential, $V_{k}^{\mathrm{lN}}(\rho)$ :

$$
\begin{aligned}
\partial_{t} V_{k}^{\mathrm{IN}}(\rho)= & \frac{k^{5}}{12 \pi^{2}}\left\{\frac{N_{\pi}}{\epsilon_{k}^{\pi}}\left(1+2 n_{B}\left(\epsilon_{k}^{\pi}\right)\right)\right. \\
& \left.-\frac{4 \times 2 \times 3}{\epsilon_{k}^{q}}\left(1-n_{f}\left(\epsilon_{k}^{q}+\mu\right)-n_{f}\left(\epsilon_{k}^{q}-\mu\right)\right)\right\}
\end{aligned}
$$

and

$$
\begin{aligned}
\partial_{t} h_{k}^{\mathrm{IN}}(\rho)= & -4 N_{\pi} v_{3}\left(h_{k}^{\mathrm{lN}}(\rho)\right)^{3} L_{(1,1)}^{(4)}\left(m_{q, k}^{2}, m_{\pi, k}^{2} ; T, \mu\right) \\
& -2 N_{\pi} v_{3} k^{2}\left(h_{k}^{\mathrm{IN}}(\rho)\right)^{\prime} l_{1}^{(B, 4)}\left(m_{\pi, k}^{2} ; T\right) .
\end{aligned}
$$

This concludes our derivation of the set of flow equations solved in the present work: The system of flows at finite $N$ is given by (5) and (11), and those in the large- $N$ limit are given in (14) and (15). Numerical results for both systems will be presented in Sec. IV, and the discontinuous Galerkin setup, with which the numerical results are achieved, is discussed in the next section.

\section{DISCONTINUOUS GALERKIN METHODS IN THE FRG}

Most of the flow equations introduced in the previous Sec. II can only be solved numerically. In the present work we use discontinuous Galerkin methods (DG methods), which have been introduced to the FRG in [67] by the example of the large- $N$ limit in an $O(N)$ model. In contrast to the set of flow equations discussed in the present work for the QM model, the flow equation for the effective potential in the large- $N$ limit of the $O(N)$ model is given by a hyperbolic equation of first order that can be written in a conservative form. This type of equation is well studied and understood. Many different numerical schemes were developed to obtain a stable solution in a weak sense; see e.g., [79].

In the present case, the flow equations (14) and (15) in the large- $N$ limit are not conservative anymore. Indeed, for a constant Yukawa coupling the QM model can be understood as a driven $O(N)$ model, where the driving force is provided by the quark loop. If this approximation to the effective action is upgraded to one with a cutoff-dependent quark two-point function, there is backcoupling effective from the meson loop into the quark loop, and the driving force is not (fully) independent anymore.

In any case the system of differential equations ceases to be conservative. For the nonconservative hyperbolic problem, like the large- $N_{f}$ equation with running Yukawa coupling, the concept of a weak solution was introduced relatively recently in $[80,81]$ and applied in a context of finite volume and discontinuous Galerkin schemes [82-89] for multiple physical systems. Hyperbolic equations in nonconservative form occur rather frequently in modeling physical systems; as an example, viscous relativistic hydrodynamic equations are of this type [90-97], and recently also a formulation of general relativity has been solved in this formulation [98] highlighting more advanced stability properties. Moreover, in the case of finite $N_{f}$ it also contains a diffusion term that originates in the $\sigma$ loop. Thus, on the technical level, the present work represents a nontrivial extension of [67]. The different extensions are discussed in Secs. III A (nonconservative systems) and III B (diffusion terms). With respect to these extensions the present work should be considered as a first step to the full implementation of DG methods for nonconservative systems; more details and further extensions will be considered elsewhere. For a more detailed introduction to DG methods see also [99].

In the context of the FRG, further work concerning the inclusion of higher-order derivatives and nonconservative formulations has been achieved in [100], which will be published soon.

Pseudospectral methods are an integral part of DG methods. They are applicable to FRG equations in the absence of shocks and have been used successfully in e.g., [101-105].

\section{A. Nonconservative flux equations}

In this section the extension of DG methods for the FRG to nonconservative flow equations is set up. To this end we consider a system of differential equations of the form

$\partial_{t} u_{i}+\partial_{\rho} f_{i}(\boldsymbol{u}, \rho, t)+a_{i j}(\boldsymbol{u}, \rho, t) \partial_{\rho} u_{j}=s_{i}(\boldsymbol{u}, \rho, t)$,

where $\boldsymbol{u}=\left(u_{1}, u_{2}\right)^{T}$ and $i, j \in\{1,2\}$. The $s_{i}$ are source terms and $f_{i}$ conservative fluxes. In (16) we also allow for nonconservative terms $a_{i}$. In the full quark-meson model 
the flux is additionally separated into a convective and a diffusive contribution depending also on $\partial_{\rho} u_{i}$. We note that the splitting into conservative and nonconservative terms is not unique in these equations. More details on the numerical treatment can be found in Appendix A, whereas an evaluation of the convergence properties of the scheme is performed in Appendix B. In the following, (5), (14) and (15) are reformulated to fit (16).

\section{Flow of the effective potential}

Equations (5) and (14) are rearranged to ensure that they have the conservative form required by (16) such that DG methods are applicable in a purely conservative formulation. Similarly to [67] we observe a nonlinear dependence of the potential $V_{k}(\rho)$ on its derivative with respect to the field expectation value $\partial_{\rho} V_{k}(\rho)$ and in the case of (5) also on its second derivative. The dependency on the first derivative can be eliminated by introducing it as a new variable, which coincides with the pion mass squared:

$$
u_{k}(\rho)=\partial_{\rho} V_{k}(\rho)=m_{\pi, k}^{2} \text {. }
$$

Since (14) is not dependent on itself we can simply take a $\rho$ derivative, which turns $u_{k}(\rho)$ into a conserved quantity which is fit for DG schemes. This procedure is also applied to the QM model. In this case we need an additional expression for the second derivative of the potential in the sigma mass in (8). We obtain this expression by taking another $\rho$ derivative of the polynomial basis functions $\phi_{n}$ introduced in Appendix A:

$$
\partial_{\rho}^{2} V(t, \rho)=\partial_{\rho} u(t, \rho)=\sum_{n=1}^{N+1} \hat{u}_{n}(t) \partial_{\rho}\left(\phi_{n}(\rho)\right) .
$$

\section{Flow of the field-dependent Yukawa coupling}

The flow of the Yukawa coupling at finite $N$ is given by a highly nonlinear equation of second order. Since it can not be made to fit the form given in (16), it is not solved within the introduced framework. However, the expression simplifies significantly in the large- $N$ limit and (15) can be written to suit the formalism. Equation (15) is rewritten in terms of the quark mass squared $m_{q}^{2}(\rho)$, as we are primarily interested in physical observables. Thus, a new variable is introduced:

$$
w_{k}(\rho)=2 h_{k}(\rho)^{2} \rho=m_{q, k}^{2} .
$$

We refer to Appendix D for the explicit calculation. Introducing this new variable proves to be very helpful for the computation. Appendix E explains how the ambiguity in splitting the conservative and nonconservative contributions to the flux are used to accommodate boundary conditions. For completeness the final form of the equations is stated:

$\partial_{t} u_{k}=\partial_{\rho} f_{u}\left(u_{k}, w_{k}\right)$,

$\partial_{t} w_{k}=\partial_{\rho}\left(a\left(u_{k}\right) w_{k}\right)-\left(w_{k} \partial_{u} a\left(u_{k}\right)\right) \partial_{\rho} u_{k}+s\left(u_{k}, w_{k}\right)$.

This version of the equation has the advantage that the nonconservative product is rather small in comparison to the conservative part.

\section{B. Finite- $N$ equations}

For finite $N$, the equation is parabolic. Apart from the convection term (Goldstones), there is also a diffusion term that arises from the $\sigma$ loop. The equations for finite $N$ are highly nonlinear and of second order; therefore, we refrain from considering the field dependency of the Yukawa coupling. Schematically the flow equation of the potential is written as

$$
\partial_{t} u_{k}=\partial_{\rho} f_{u}\left(u_{k}, w_{k}, \partial_{\rho} u_{k}\right)
$$

The weak formulation and the stability of this type of equation have not been fully understood until now. The presence of the diffusion modifies the numerical flux significantly. However, in the convection-dominated regime, and in the absence of a discontinuity, it is possible to neglect this diffusion numerical flux and formulate the discontinuous Galerkin method for this equation as follows:

$$
\begin{gathered}
\int_{D^{k}}\left(\left(\partial_{t} u_{i, h}+a_{i, h} \partial_{\rho} u_{i, h}+s_{i, h}\right) q_{n}+f_{i, h} \partial_{x} q_{n}\right) d x \\
=-\int_{\partial D^{k}} q_{n}\left(f_{i}^{*} \hat{\mathbf{n}}+\mathbf{D}\left(u_{i, h}^{+}, u_{i, h}^{-}, \hat{\mathbf{n}}\right)\right) d x,
\end{gathered}
$$

where $a_{i, h}, s_{i, h}$ and $f_{i, h}$ are computed form the field $u_{i}$ and their local approximation of the derivative; no other numerical fluxes are introduced into the numerical scheme. The absence of numerical fluxes for the extra derivative present in the equation corresponds to the assumption of continuity of this field, and the DG scheme somehow reduces to a pseudospectral method. This approximation is acceptable, whenever the flow is rather smooth and no shock or rarefaction wave are generated during the simulation. In turn, this scheme will fail in the vicinity of a firstorder phase transition. There we expect shock formation and propagation in the flow equation. In conclusion, for the rest of the phase diagram the present approximation can be considered as a sufficiently accurate solution of the flow equation due to the local high-order accuracy of the DG scheme. 


\section{RESULTS}

In this section we present and discuss our results for the phase structure of the QM model in the different approximations. We chose our initial conditions such that the quark mass and the pion decay constant reproduce physical values in the vacuum; this is discussed in detail in Sec. IV A. Note, however, that it is not the main objective of the present work to produce quantitatively reliable results; the vacuum benchmarking simply facilitates the understanding of our results. In the present work we rather focus on the qualitative behavior of the matter sector of QCD at large densities; quantitatively reliable results require full QCD flows and will be considered elsewhere. Such a setup entails that, while we compute and present a phase structure at large densities, our present low-energy effective theory gradually loses predictability for larger $\mu_{B} / T$. Such an estimate in functional QCD leads to a predictability bound of $\mu_{B} / T \lesssim 4$, if the currently existing state of the art computations are combined $[12,23,24]$ and estimates for missing channels and effects are considered as well $[12,14,106]$. In the present class of low-energy effective theories (QM, Nambu Jona Lasino type, PQM, PNJL), a respective estimate leads to $\mu_{B} / T \lesssim 2$.

We first present results within an approximation where only the effective potential depends on the cutoff scale, the local potential approximation (LPA), for both the finite- $N_{f}$ and the large- $N_{f}$ limit in Sec. IV B. They serve as a benchmark for the more advanced approximations discussed in Sec. IV C. Additionally, the results in Sec. IV B also serve as a benchmark for results in the literature within the QM and Polyakov loop-enhanced QM models, in particular at large density, where DG methods or similar numerical approaches are mandatory for reliable results.

In Sec. IV C we present results for the coupled system of effective potential $V_{k}(\rho)$ and Yukawa coupling $h_{k}(\rho)$ in the large- $N_{f}$ limit. This investigation allows us to solidify the results in [47] concerning the flattening of the quark mass $m_{q}(\phi)$.

Lastly, the technical advances made here readily carry over to first principle QCD within the FRG; as discussed in the introduction, they are one of two missing ingredients for reliable predictions of the QCD phase structure for $\mu_{B} / T \gtrsim 4$.

\section{A. Initial conditions}

We initiate the flow at a cutoff scale $k=\Lambda \approx 0.650 \mathrm{GeV}$ with the classical action of the QM model. Then, the parameter in the initial effective action $\Gamma_{\Lambda}$ is the $\phi^{4}$ coupling in the classical potential:

$$
u_{\Lambda}(\rho)=\frac{\lambda_{\Lambda}}{2} \rho, \quad w_{\Lambda}(\rho)=2 h_{\Lambda}^{2} \rho,
$$

as well as the Yukawa coupling $h_{\Lambda}$. For the sake of simplicity we use a initial meson quark mass $m_{\phi}^{2}=0$.
TABLE I. Low-energy observables and related EFT couplings at the initial cutoff scale $\Lambda_{\mathrm{UV}}=0.65 \mathrm{GeV}$. The scales are fixed with the pion decay constant in the chiral limit $f_{\pi, \chi}=88 \mathrm{MeV}$, that is, $m_{\sigma} / f_{\pi, \chi} \approx 3.603$ and $m_{q} / f_{\pi, \chi} \approx 3.532$. In the present approximation we have $f_{\pi, \chi}=\sigma_{0}$, and in the model the dimensionless ratios are simply $m_{\sigma} / \sigma_{0}$ and $m_{q} / \sigma_{0}$. In the chiral limit we also have $m_{\pi}=0$.

\begin{tabular}{lcc}
\hline \hline Observable & Value $[\mathrm{MeV}]$ & Parameter at $\Lambda_{\mathrm{UV}}=0.65 \mathrm{GeV}$ \\
\hline$m_{\sigma}$ & 317.1 & $\lambda_{\Lambda}=71.6$ \\
$m_{q}$ & 310.8 & $h_{\phi, \Lambda}=3.6$ \\
\hline \hline
\end{tabular}

All our scales are measured in the pion decay constant in the chiral limit $f_{\pi, \chi}=88 \mathrm{MeV}$. Within the present approximation of the QM model we have $f_{\pi} \approx \sigma_{0}$, and hence we define $\sigma_{0}=88 \mathrm{MeV}$. Then, the two model parameters $\lambda_{\Lambda}$ and $h_{\Lambda}$ are fixed such that they lead to a "physical" constituent quark mass $1 / \sqrt{2} h \sigma_{0}$ and a physical mass of the sigma resonance, $m_{\sigma}$. The parameters for the couplings of the effective theory and their relation to physical observables are summarized in Table I. The dimensionless ratios in the models at $k=0$ are given by

$$
\frac{m_{\sigma}}{\sigma_{0}} \approx 3.605, \quad \frac{m_{q}}{\sigma_{0}} \approx 3.532
$$

and follow with the initial parameters in Table I.

\section{B. Results for the effective potential with constant Yukawa coupling}

In this section we compare the numerical results of the physical case with $N_{f}=2$ and in the large- $N_{f}$ limit with three and four degrees of freedom. This is done in LPA, where we solve the flow equation for the effective potential (14). We first discuss the asymptotic regimes: vacuum, large temperatures, and large chemical potential in Sec. IV B 1. Then we show that the chiral phase transition, or rather its nonuniversal properties, agrees quantitatively for all models in Sec. IV B 2. The shock development at large chemical potential is discussed in Sec. IV B 3. Finally, we compare the phase structure for all three cases in Sec. IV B 4.

\section{Asymptotic regimes}

We begin with an evaluation of the asymptotic regimes: the vacuum with $\mu_{q}, T=0$, large temperatures with $\mu_{q}=0$, and large chemical potentials with $T=0$. For these cases we show the field dependence of the pion mass $m_{\pi, k}(\rho)=\partial_{\rho} V_{k}(\rho)$; see (17) for different cutoff scales. This resolves the effective potential, obtained from an integration over $\rho$, in terms of a physical observable.

For the numerical solution of (14) we use an interval of $\rho \in[0,0.02] \mathrm{GeV}$, which is expanded in $K=80$ cells with polynomials of order $N_{p}=2$. The length of the interval is 


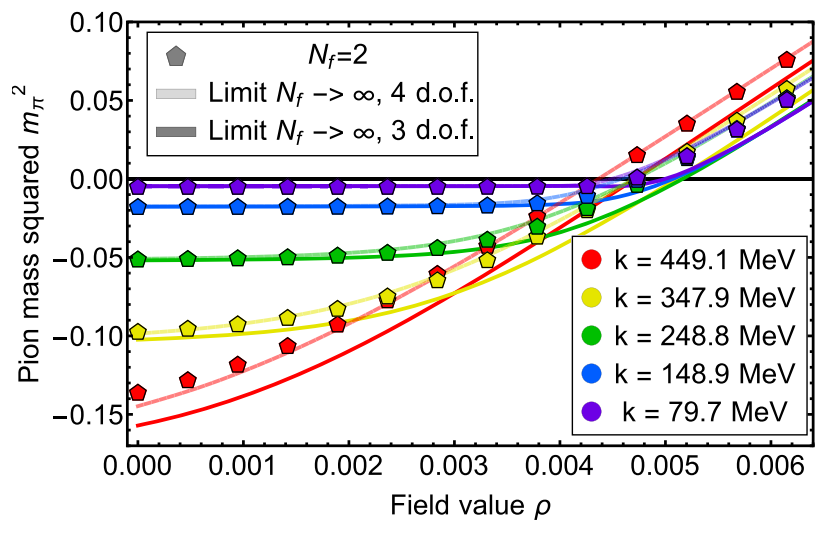

FIG. 2. RG-scale evolution of the field-dependent pion mass $m_{\pi}^{2}(\rho)$ in LPA (constant Yukawa coupling) in the vacuum. We compare results for $N_{f}=2$ and the large- $N_{f}$ limit with three and four degrees of freedom; see Sec. II C.

chosen such that it includes all relevant phenomena: The flux at the outer boundary is very small. This ensures the numerical convergence for the entire phase diagram.

The benchmark case is the vacuum, where the present models are anchored; see Sec. IV A. The field dependence of the pion mass is shown in Fig. 2. For the initial cutoff $k=\Lambda$, the pion mass is simply a straight line, $m_{\pi, \Lambda}(\rho)=\lambda_{\Lambda} \rho$, where the slope is the initial mesonic self-coupling $\lambda_{\Lambda}$. With decreasing cutoff scale, the pion mass develops a flat regime, which is related to the emergence of nontrivial minima $\rho_{0}=\sigma_{0} / 2$ in the potential, indicating chiral symmetry breaking.

We also conclude, from the comparison of the pion masses in the different models, that the cutoff dependence of the physical two-flavor case is best mimicked by the large- $N_{f}$ limit with four degrees of freedom: Most of the dynamics takes place in the symmetric regime or close to it. Technically, this regime is described with $1+m_{\sigma}^{2}\left(\rho_{0}\right) / k^{2} \approx 1+m_{\pi}^{2}\left(\rho_{0}\right) / k^{2}$, owing to the fact that the total mass of the respective modes is $k^{2}+m_{\pi / \sigma}^{2}$.

Finally, we determine the relative values of the pion decay constants in the chiral limit, $f_{\pi, \chi}=\sigma_{0}$, in the different models. All scales are measured in the pion decay constant $f_{\pi}=88$ in the two-flavor case. The expectation value $\sigma_{0}$ or $\rho_{0}=\sigma_{0}^{2} / 2$ follows from (7) as the maximal field value with $m_{\pi}\left(\rho_{0}\right)=0$. However, since we stop the numerics at a small but finite cutoff value $k_{\min }=50 \mathrm{MeV}$, we extrapolate the expectation value $\sigma_{0}\left(k_{\min }\right)$ to $\sigma_{0}(0)$ within a linear fit: We use data from ten equally spaced RG scales from $k=$ $90 \mathrm{MeV}$ to $k=50 \mathrm{MeV}$ and fit

$$
\sigma_{0, k}=\sigma_{0}+\text { const } k
$$

The self-consistency of this linear fit is checked by the perfect agreement of the linear fit with the data; see Fig. 3.

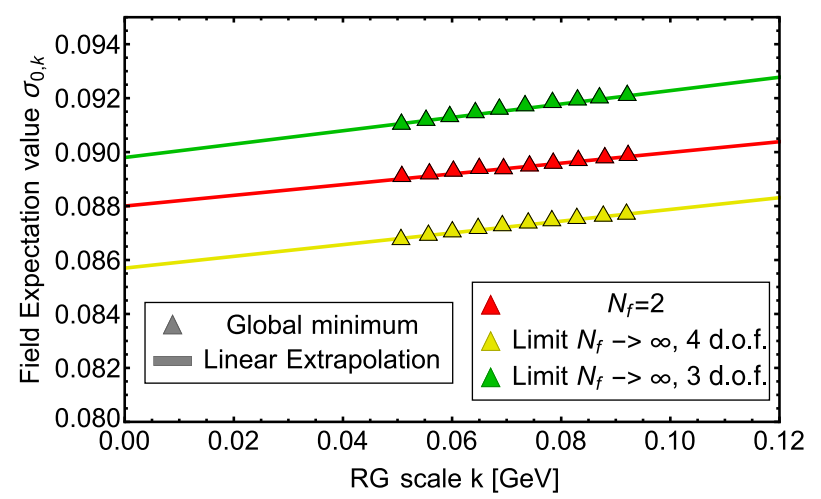

FIG. 3. Linear extrapolation of the expectation value $\sigma_{0}$. The corresponding fit parameters to (25) can be found in Table II.

The respective values for $\sigma_{0}$ are given in Table II. The mass $m_{\sigma}$ of the scalar mode is extrapolated to $k=0$ from the same data. Note, however, that once the kink enters the cell in which $\sigma_{0}$ is located, the precise determination of the derivative is difficult. Therefore, the flattening of the potential most likely causes an underestimation of $m_{\sigma}$.

For large temperatures we safely stay in the symmetric regime and the mesons simply acquire a thermal Debye mass. This is seen in Fig. 4(a). In the symmetric regime we have four mesonic degrees of freedom in the two-flavor case, consistent with our expectations, that the cutoff dependence of the pion mass in the model with four degrees of freedom in the large- $N_{f}$ limit has the best agreement with the two-flavor case.

We close with a discussion of the large chemical potential asymptotics. The respective pion mass (squared) is depicted in Fig. 4(b). The sudden increase of the pion mass for $k \lesssim k_{\text {on }}$ with $k_{\text {on }} \approx \mu$ in Fig. 4(b) is related to the Silver-Blaze property [107]; for the discussion in the FRG approach see $[50,108,109]$. This property entails that correlation functions below the density onset are simply functions of $p_{0} \mp i \mu_{q}$ for quark and antiquark frequencies, respectively. Accordingly, observables do not depend on the chemical potential for $\mu_{q}<\mu_{q, \text { on }}$, where $\mu_{q, \text { on }}$ is the onset chemical potential. For $\mu_{q}>\mu_{q \text {,on }}$, the medium leads to deformations of the quark-meson scattering processes,

TABLE II. Expectation value $\sigma_{0}$ for the three models obtained by a fit of (25) to the zero point at five equally spaced RG scales $k=90 \mathrm{MeV}$ to $k=50 \mathrm{MeV}$. The error is computed from the grid resolution and the error to the fit parameters. The mass $m_{\sigma}$ is extrapolated from the same data points as the fit. The error of $m_{\sigma}$ is obtained analogously to the one of $\sigma_{0}$; it might be underestimated due to the kink developing at $\sigma_{0}$.

\begin{tabular}{lccc}
\hline \hline & $\sigma_{0}[\mathrm{MeV}]$ & const & $m_{\sigma}[\mathrm{MeV}]$ \\
\hline$N_{f} \rightarrow \infty, 3$ d.o.f. & $89.8(17)$ & $0.0755(32)$ & $335(15)$ \\
$N_{f} \rightarrow \infty, 4$ d.o.f. & $85.7(18)$ & $0.0752(35)$ & $311(10)$ \\
$N_{f}=2$ & $88.0(20)$ & $0.0360(26)$ & $317(12)$ \\
\hline \hline
\end{tabular}



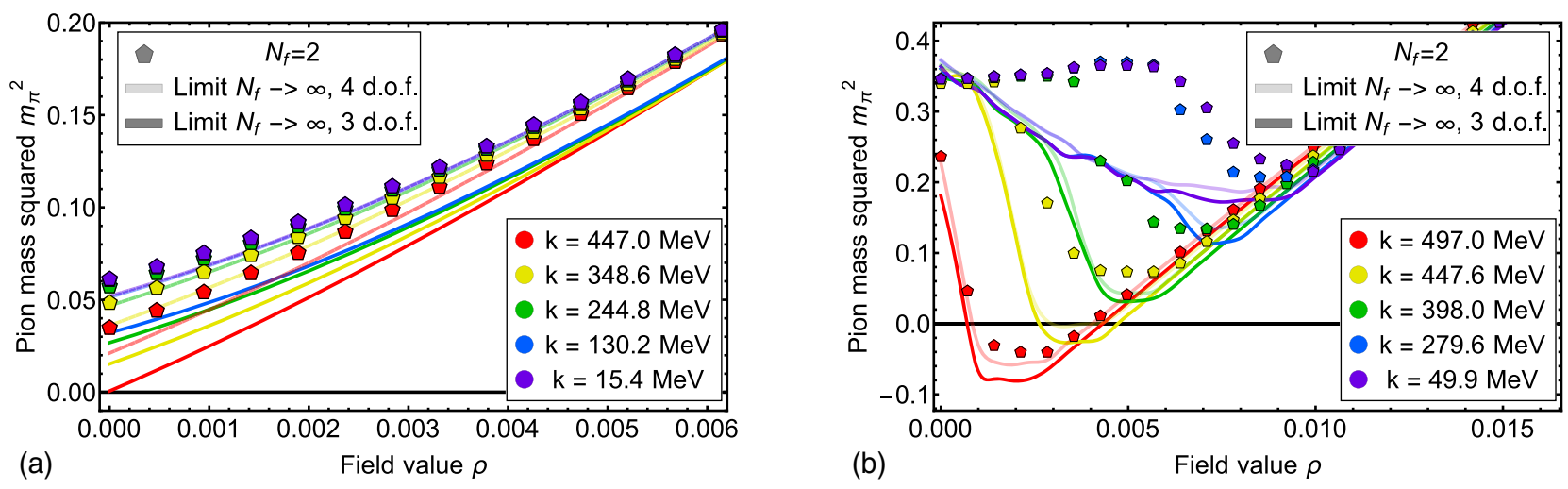

FIG. 4. RG-scale evolution of the field-dependent pion mass $m_{\pi}^{2}$ in the symmetric phase. In this figure we compare results for the finite- $N_{f}$ case and the large- $N_{f}$ limit with three and four degrees of freedom, respectively. The Yukawa coupling is kept at a constant value. (a) Pion mass at zero density and high temperature $(T=280 \mathrm{MeV})$. The flow is washed out by the temperature fluctuations. (b) Pion mass at zero temperature and high density $(T=500 \mathrm{MeV})$. The density onset introduces a sharp edge to the flow.

comprised in medium meson dispersions. In the presence of thermal fluctuations this onset is washed out with increasing temperature.

Note also that the onset cutoff scale depends on the field value, as in the present approximation the onset chemical potential is given by $\mu_{q, \text { on }}^{2}=1+m_{q, k}^{2}$.

In summary, the results in the asymptotic regimes show the expected physics phenomena. Moreover, the comparison of the large- $N_{f}$ models with the two-flavor case quantifies the similarities between the large- $N_{f}$ limit models and the physical two-flavor model.

\section{Chiral phase transition at vanishing density}

These similarities are furthered by a study of the chiral phase transition at vanishing density, $\mu_{q}=0$. In particular we present a detailed comparison of the temperature dependence of the chiral order parameter $\sigma_{0}$ in the

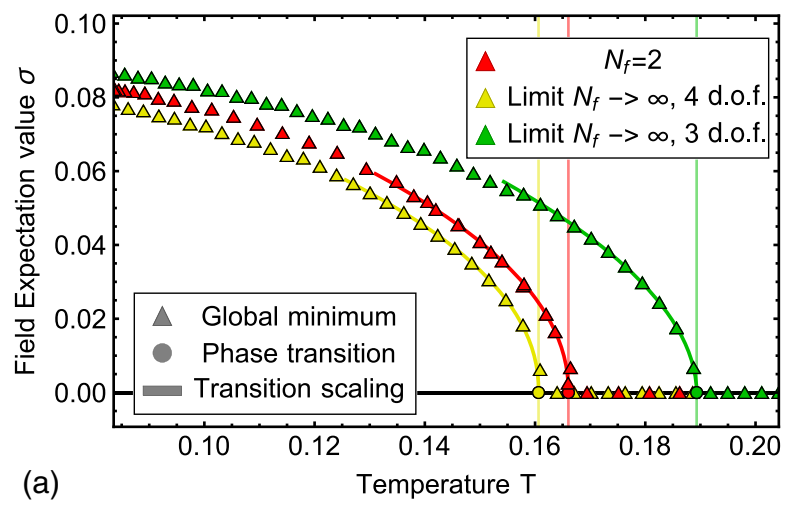

$N_{f}=2$-flavor case with the large- $N_{f}$ limits with three and four degrees of freedom. The numerical results for $\sigma_{0}(T)$ are displayed in Figs. 5(a) and 5(b).

In these computations we use a grid $\rho=[0,0.2] \mathrm{GeV}$ and expand in $K=100$ elements with a local approximation order of $N_{p}=2$, and the $\sigma$ is obtained by a linear extrapolation as described in Sec. IV B. The data in proximity of the transition point are compatible with the scaling law

$$
\sigma(T)= \begin{cases}c_{\text {cr }}\left|T-T_{\text {crit }}\right|^{\beta}, & T \leq T_{\text {crit }}, \\ 0, & T \geq T_{\text {crit }},\end{cases}
$$

where for $N_{F} \rightarrow \infty$ we have $\beta=1 / 2$, the mean-field critical exponent. In turn, for the present LPA study of the Yukawa model with the $\mathrm{O}(4)$-universality class we have used the three-dimensional spatial flat or Litim regulator [78] for both

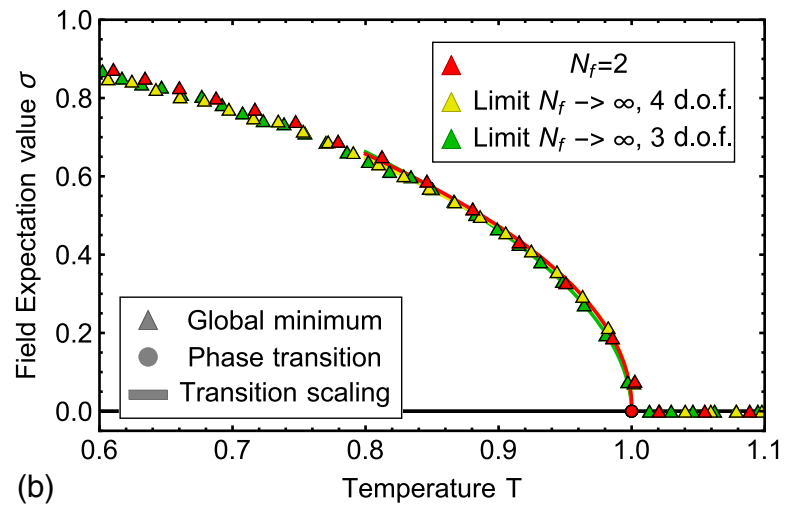

FIG. 5. Temperature dependence of the mesonic field expectation value $\sigma_{0}$. The figure shows a second-order phase transition at fixed chemical potential $\mu=0 \mathrm{GeV}$. We compare the case for finite $N_{f}$ and the large- $N$ limit with three and four degrees of freedom with a constant Yukawa coupling. Fitting (26) to the data gives values for the critical temperatures and exponents of the transition, with the parameters given in Table III. Deviations to the fit values are due to the low resolution in the extrapolation of the zero point on the numerical grid. (a) Absolute values of thefield expectation value $\sigma$. Units are given in GeV. (b) Axes rescaled by the respective expectation values and critical temperatures. 
quarks and mesons. See also Appendix H, (H1) and (H2). This leads us to $\beta \approx 0.40$; see [110] with [111,112] and in particular the recent work in the QM model [19]. Note that more advanced approximations of the FRG provide $\beta \approx 0.39$ consistent with conformal bootstrap and Monte Carlo results. For a recent compilation see [113]; for the QM model see [19] that also includes an investigation of the $Z_{2}$-universality class.

The respective scaling regimes are already very small in the $O(4)$ model and even shrink in the presence of the (driving) fermion loop; see the discussion in [19]. While possible, we do not aim at a precision estimate of critical exponents here, as we focus on the location of the phase boundaries. Accordingly, we have simply checked the consistency of the scaling law (26) with $\beta \approx 0.4$ $\left(N_{f}=2\right)$ and $\beta=1 / 2\left(N_{f} \rightarrow \infty\right)$ for small reduced temperatures $1-T / T_{c} \rightarrow 0_{-}$. This also allows us to determine the respective scaling regimes. Consistent with the observation above that they should be even smaller as the already small scaling regime in $O(N)$ models we find scaling for

$$
0<1-T / T_{c} \lesssim 10^{-2} .
$$

Moreover, a scaling fit with (26) in the regime (27) allows us to determine $T_{c}$ as well as the prefactor $c_{\mathrm{cr}}$.

We see from Table III that the two-flavor critical temperature agrees well with the large- $N_{f}$ limit with four degrees of freedom. This is expected from the theoretical analysis and our results on the asymptotics in Sec. IV B 1. This good agreement extends to the full temperature dependence, as can be seen from Fig. 5(a). In turn, the order parameter from the large- $N_{f}$ limit with three degrees of freedom seemingly shows a slightly different behavior.

However, the two large- $N_{f}$ models are obtained by a simple rescaling of the fields and hence are identical to each other. They can be mapped onto each other by the relative rescaling. Put differently, the temperature dependence of the order parameters should agree if plotted in dimensionless units, $\sigma(T) / \sigma(0)$ and $T / T_{c}$. This comparison is shown in Fig. 5(b): As expected, the temperature dependences of the order parameter of the large- $N_{f}$ models agree. More importantly, also the two-flavor case agrees quantitatively, though with small deviations. Trivially, the nontrivial

TABLE III. Parameters obtained from a $\chi^{2}$ fit of (26) to the mean-field expectation values in Fig. 5(a) which are underlined by the transition scaling. The error in the data is expected to be higher, since the numerical precision is limited by the grid resolution. An exact reconstruction of the zero crossing is not possible.

\begin{tabular}{lcc}
\hline \hline Model & $c_{\text {cr }}$ & $T_{\text {crit }}$ \\
\hline$N_{f} \rightarrow \infty:$ : 3 d.o.f. & $0.2985(24)$ & $0.18929(11)$ \\
$N_{f} \rightarrow \infty: 4$ d.o.f. & $0.3010(33)$ & $0.16139(10)$ \\
Finite $N_{f}$ & $0.2126(61)$ & $0.16618(19)$ \\
\hline \hline
\end{tabular}

critical scaling of the two-flavor case does not agree with the trivial mean-field scaling for $N_{f} \rightarrow \infty$, but the scaling regimes are very small; see (27).

In summary, the thermal properties of the models in the large- $N_{f}$ limit and the physics case $N_{f}=2$ agree very impressively.

\section{Shock development and first-order phase transition at high densities}

In this section we discuss the shock development and propagation at intermediate densities and very low temperatures. This is also used to discuss the first-order regime.

In these computations we use a grid $\rho=[0,0.2] \mathrm{GeV}$ and expand in $K=200$ elements with a local approximation order of $N_{p}=2$. This finer grid is required for the shock resolution at low temperatures and chemical potentials close to the onset chemical potential. Indeed, the full resolution of some of the features in this regime (e.g., the precise location of the transition line in the absence of shocks) requires an even higher resolution. While technically possible, we have refrained from doing so, as the related aspects have been not in the main focus of the present work.

We first note that the running of the pion mass stops quickly below the onset RG time $t_{\text {on }}=\ln \frac{\Lambda}{\mu}$ : The RG flow is proportional to the Fermi distribution. Hence it stops at $k_{\text {on }}$ at $T=0$; for finite $T$ the Fermi distribution is softer, but for small temperatures there is still a strong exponential suppression for $k \leq k_{\text {on }}$. This suppression leads to two competing effects at finite densities.

(i) At high densities the suppression of the quark contribution creates positive meson masses $m_{\pi, k}^{2}(\rho)$ and determines the value of $m_{\pi}^{2}\left(\rho_{0}\right)$ in the symmetric phase [compare to Fig. 4(b)]. The quark contribution dominates initially, but due to the constant Yukawa coupling it is quickly suppressed with $k^{5}$, decreasing the effect of the suppression at lower densities.

(ii) For field values with positive meson masses $m_{\pi, k}^{2}(\rho)$ the meson loop in the flow is suppressed with $k^{5}$. In turn, for negative meson masses the meson loop is suppressed with $k^{4}$. Note also that the flow increases with decreasing values of $m_{\pi, k}^{2}$, which is closely linked to the restoration of convexity. The mesonic flow contribution is reminiscent of the spreading of waves in hydrodynamics, where its value corresponds to the wave velocity: If we consider the solution $m_{\pi}^{2}(\rho)$ as a wave packet, it flows with the RG time in the direction of smaller field values with a $\rho$-dependent propagation velocity. The velocity of the solution and its effect on convexity is inspected more closely in Appendix C.

The interplay of both effects leads to the creation of shocks and a first-order phase transition at low temperatures. The increased propagation speed of negative modes is blocked 
by the slowed propagation of positive modes. The shock travels toward smaller field values during the RG-time evolution but eventually freezes when the shock amplitude is too high. An illustration of this process can be found in Appendix F.

Naturally, the occurrence of shock development depends on the choice of initial conditions, specifically those that trigger stronger dynamics of the system; for a respective discussion in the $O(1)$ model see [67]. With physical initial conditions we find shock development in the large- $N_{f}$ limit with three d.o.f., whereas the dynamics for four d.o.f. are not strong enough to generate a shock at finite temperature $T>10 \mathrm{MeV}$. This is an important observation: We have used the same initial conditions for all models, fixed within the two-flavor case. As discussed before, the two models in the large- $N_{f}$ limit only differ by a rescaling of the fields and parameters. Accordingly, they can be interpreted as the same model with different initial conditions, as we do not apply any rescaling to the initial condition. However, these changes are marginal, as can be seen from the small variation of the pion decay constants and $\sigma$ masses in the vacuum; see Table II. In conclusion the physical case is very close to the situation where shocks may form during the RG-time evolution. Whether or not this also occurs in QCD requires further investigation.

(i) The embedding of the present model as part of the matter sector will lead to additional driving forces in the flow. This may be mimicked with a $T, \mu$ dependent change of the initial conditions here. Naturally these changes can go either way; they may support the shock development or soften it.

(ii) The additional diffusion terms in the finite- $N_{f}$ case (see Sec. III B) may structurally soften the RG-time evolution and remove any shock development. It is also unclear whether shocks develop in the presence of diffusion terms allow, as the diffusive flux counteracts the formation of a discontinuity.

The resolution of these aspects is crucial for an access to the QCD phase boundary at large chemical potential and low temperatures. This goes far beyond the scope of the present work and is subject of ongoing work.

In the large- $N_{f}$ limit with three d.o.f., we can use the shock development at low temperatures for an accurate determination of the phase transition line. The shock position $\xi_{\text {final }}$ at $k=0$ is extrapolated by fit, utilizing the exponential decay of the flows:

TABLE IV. Parameters obtained from a $\chi^{2}$ fit of (28) to the shock positions in Fig. 6.

\begin{tabular}{lccc}
\hline \hline Parameter & $\begin{array}{c}\text { Prefactor } \\
\beta\end{array}$ & $\begin{array}{c}\text { Crit. exponent } \\
\zeta\end{array}$ & $\begin{array}{c}\text { Crit. density } \\
\mu_{\text {crit }}\end{array}$ \\
\hline$\chi^{2}$ fit & $0.391(45)$ & $0.524(31)$ & $0.30460(33)$ \\
\hline \hline
\end{tabular}

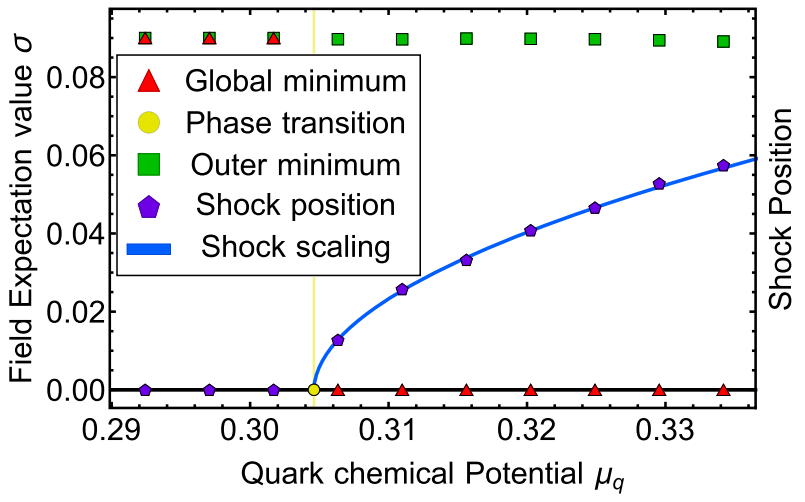

FIG. 6. Density dependence of the mesonic field expectation value $\sigma_{0}$ at $T \approx 0$ for the large- $N_{f}$ model with three d.o.f. in LPA (constant Yukawa coupling). The figure shows a first-order phase transition of the field expectation value at zero temperature. The solution now contains a local outer minimum and shock development between the outer local and inner global minimum. The extrapolated shock position at $k=0 \mathrm{GeV}$ is plotted at different densities. We find that the shock position, in the vicinity of the first-order transition, obeys the scaling law in Eq. (28). The parameters of a scaling fit [see (28)] are given in Table IV.

$$
\xi(t)=\xi_{\text {final }}+\text { const } e^{-t} \text {. }
$$

We use the shock position at six equally spaced time steps between RG times $t=3$ and $t=3.5$. We expect the same power law behavior as in [67] for the final shock position as a function of chemical potential:

$$
\xi_{\text {final }}= \begin{cases}\beta\left|\mu-\mu_{\text {crit }}\right|^{\zeta}, & \mu \geq \mu_{\text {crit }} \\ 0, & \mu \leq \mu_{\text {crit }}\end{cases}
$$

As an explicit example we concentrate on $T=10 \mathrm{MeV}$. The coefficients of the $\chi^{2}$ fit are provided in Table IV. Next to the first-order phase transition at small temperatures the position of the shock as a function of the control parameter, the chemical potential, is well approximated by the function in Eq. (28). This scaling form is the same used to describe the order parameter next to a second-order phase transition. In this sense the shock position behaves like an order parameter of a second-order phase transition when the system experiences a first-order phase transition. From the scaling form of the shock position [Eq. (28)] we obtain an accurate estimate for the critical chemical potential of $\mu_{\text {crit }}=0.30460 \pm 0.00033 \mathrm{GeV}$. The phase transition and shock positions are depicted in Fig. 6. Shock formation occurs only in a relatively small area of the $(T, \mu)$ plane, being confined to intermediate densities $290 \mathrm{MeV}<$ $360 \mathrm{MeV}$ and small temperatures up to $T=20 \mathrm{MeV}$.

In the absence of a shock a very fine grid has to be used to pin down the phase transition line for low temperatures. In the present work we have simply narrowed down the 

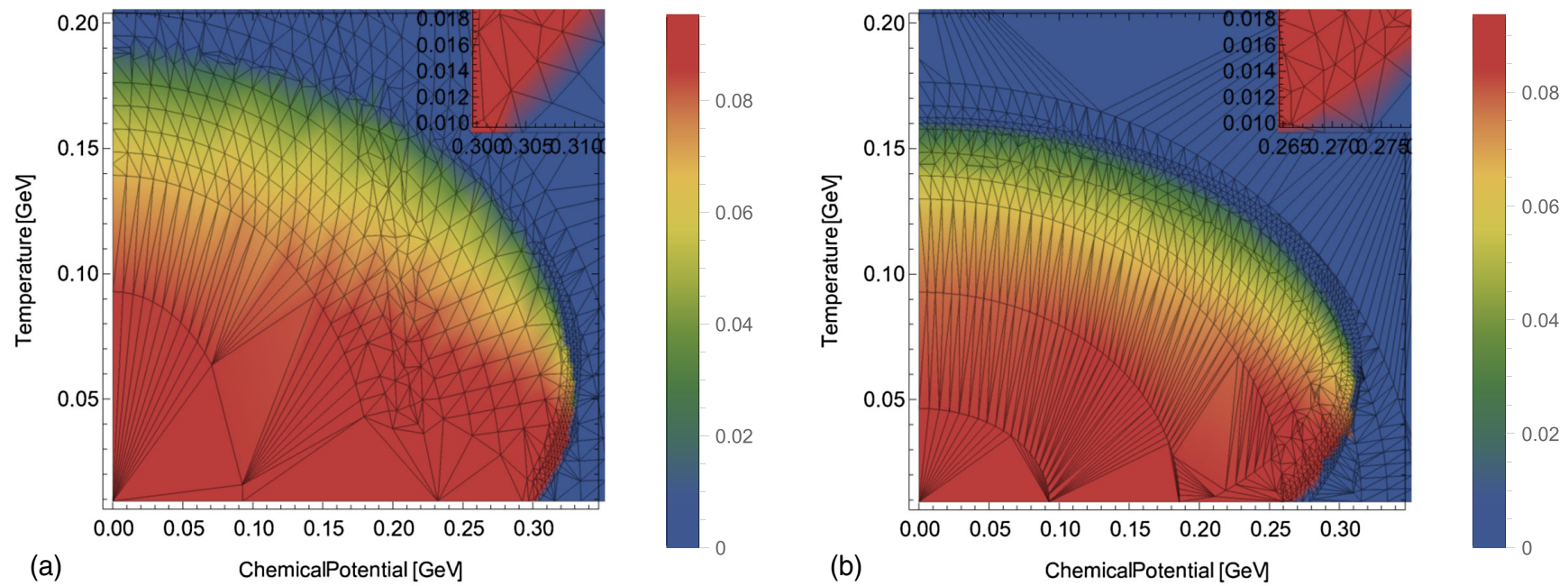

FIG. 7. Phase diagram of the large- $N$ model in the $(T, \mu)$ plane with constant Yukawa coupling, where the mean-field expectation value $\sigma$ is chosen as order parameter. The mesh indicates the discrete data points. (a) Large $N_{f}$ with 3 DoF, with slight shock development at high densities. (b) Large $N_{f}$ with 4 DoF and no shock development at high densities.

location of the phase transition line for small temperatures $T \lesssim 30 \mathrm{MeV}$ to a small interval $\mu_{\text {crit }} \in[270,290] \mathrm{MeV}$.

\section{Phase structure in LPA}

The preparations and results discussed in the last sections allow us to compute the phase structure of the QM model, both for $N_{f}=2$ and in the large- $N_{f}$ limit.

In these computations we use a grid $\rho=[0,0.03] \mathrm{GeV}$ and expand in $K=70$ elements with a local approximation order of $N_{p}=2$. This setup ensure convergence of the numerics for all temperatures up to $T=0.3 \mathrm{GeV}$ and chemical potentials around $\mu=0.35 \mathrm{GeV}$.

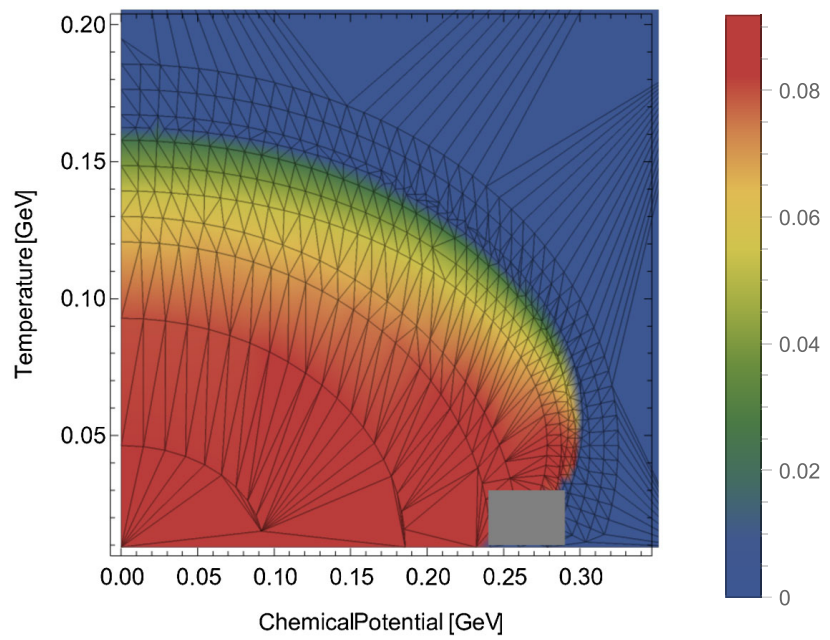

FIG. 8. Phase diagram at $N_{f}=2$ with constant Yukawa coupling. The gray box indicates the points that did not converge. A thorough discussion of the convergence issues is provided in Appendix B 1.
For the resolution of shock formation a finer grid is required. We observe a formation of shocks around the first-order phase transition in the large- $N$ case at densities around $\mu=0.3 \mathrm{GeV}$. In this area we expand in $K=250$ cells to reduce oscillations and ensure numerical convergence. The flow is evaluated up to $t=4$, which corresponds to a momentum scale of $k=0.001 \mathrm{GeV}$. The field expectation value $\sigma$ is chosen as order parameter and evaluated as demonstrated previously for the approximate vacuum.

The result for the large- $N$ limit is depicted in Fig. 7. The crossover region is discernible by the color gradient that smoothly transitions between both phases, whereas in the first-order regime a jump is clearly visible. Figure 7(b) also illustrates how shock development shifts the critical chemical potential to higher values.

The phase diagram for the finite- $N_{f}$ case is given in Fig. 8-the computation did not converge at high densities; this is further discussed in Appendix B 1. As discussed before, it will be interesting to see how this regime changes, if the present model is embedded as part of the matter sector of QCD in full QCD flows. This is subject of ongoing work.

\section{Quark-meson scatterings in the large- $N$ limit}

The discussion of the results in LPA with a constant Yukawa coupling has revealed a very intricate structure at about onset chemical potentials and low temperatures. In particular the occurrence of shocks is very sensitive to the details of the dynamics. Moreover, we expect quark-meson scattering also being important in the vicinity of a potential critical end point in QCD.

We now present our results for the QM model with a field-dependent Yukawa coupling in the large- $N_{f}$ limit with 



FIG. 9. RG-scale evolution of the field-dependent quark and pion masses in approximate vacuum in the large- $N_{f}$ limit with four d.o.f. (a) Comparision of the RG-running of the pion mass with constant and field dependent Yukawa coupling. (b) Field-dependence of the quark mass. A flat regime emerges for $k \rightarrow 0$, related to that in the effective potenial.

four d.o.f., based on the combined numerical solution of the flows (14) and (15), as formulated in (20).

\section{Dynamics in the vacuum}

For the discussion of the dynamics in the vacuum, (20) is solved on a grid with varying cell sizes. A local approximation order of $N_{p}=3$ is used with $K=100$ cells in $\rho \in[0,0.02]$. Figure 9 depicts the solutions of the pion and quark masses in approximate vacuum, the pion mass in comparison to the case with constant Yukawa coupling. We can see that in approximate vacuum the pion mass remains unchanged for both models. An exponential fit is performed on the position of the zero point of $\partial_{\rho} V_{k}(\rho)$ using five equidistant RG scales from $k=65 \mathrm{MeV}$ to $k=25 \mathrm{MeV}$, and we obtain

$$
\sigma_{0, \pi}=87.4(17) \mathrm{MeV} .
$$

This is consistent with the results in the previous sections with a constant Yukawa coupling (LPA). Consequently, this confirms previous findings in [47], that LPA or rather higher orders in the derivative expansion are a good approximation for vacuum QCD.

Figure 9(b) depicts the field-dependent quark mass $m_{q, k}^{2}(\rho)$ for different RG times. As argued in [47], the quark mass flattens for meson fields $\rho \leq \rho_{0}$. The computation in the present work puts these conceptual and preliminary numerical findings on a sound numerical footing. In summary, at vanishing cutoff scale $k=0$, this leaves us with a field-dependent quark mass $m_{q}^{2}(\rho)$ with

$$
m_{q}^{2}(\rho) \geq m_{q}^{2}\left(\rho_{0}\right)
$$

Note that while conceptually the field value $\rho_{0, q}$, below which the mass function flattens, has to agree with $\rho_{0}$, the solution of the equation of motion, numerically this is not fully guaranteed. Hence, this provides a further consistency check of the present scheme. For performing the respective reliability check, we have determined the position of the kink by subtracting $-\rho$ from the solution and taking the minimum. An exponential fit gives

$$
\sigma_{0, q}=86.0(17) \mathrm{MeV},
$$

which coincides within its error $\sigma_{0}$ in (29). The error is computed from the grid resolution and the error to the fit parameters.

The quark mass in the flattened area in Fig. 9(b) is computed as follows: One computes the average value of the quark mass up to the kink for the previously mentioned five RG scales and performs an exponential fit. This leads to the physical quark mass

$$
m_{q}\left(\rho_{0}\right)=309.635(85) \mathrm{MeV} .
$$

The linear $\rho$ dependence of $m_{q}^{2}(\rho)$ in Fig. 9(b) for $\rho>\rho_{0}$ entails that the Yukawa coupling is constant with $\partial_{\rho} h(\rho) \approx 0$, already observed in [47] for finite $N_{f}$. The constant approximation for these field values works so well that we can use the input Yukawa coupling $h_{\Lambda}$ to confirm $\sigma_{0}$ with the consistency relation $\sigma_{0}=m_{q}\left(\sigma_{0}\right) / h_{\Lambda}$. This leads us to $\sigma_{0, q^{\prime}}=86.01$ (24) $\mathrm{MeV}$, where we take the error from the fit and the mean deviation in the flattened region.

In summary the present numerical analysis confirms quantitatively the conceptual and preliminary numerical analysis in [47]: In the broken phase the flattening out of the field-dependent quark mass $m_{q}^{2}(\rho)$ is triggered by the flattening of the effective potential. In turn, in the symmetric phase the field-dependent quark mass does not flatten out and the quark mass vanishes on the solution of the equation of motion, $\rho_{0}=0$. Respective plots of the field-dependent quark mass $m_{q}^{2}(\rho)$ and the pion mass $m_{\pi}^{2}(\rho)$ for high temperature and density values are discussed in 


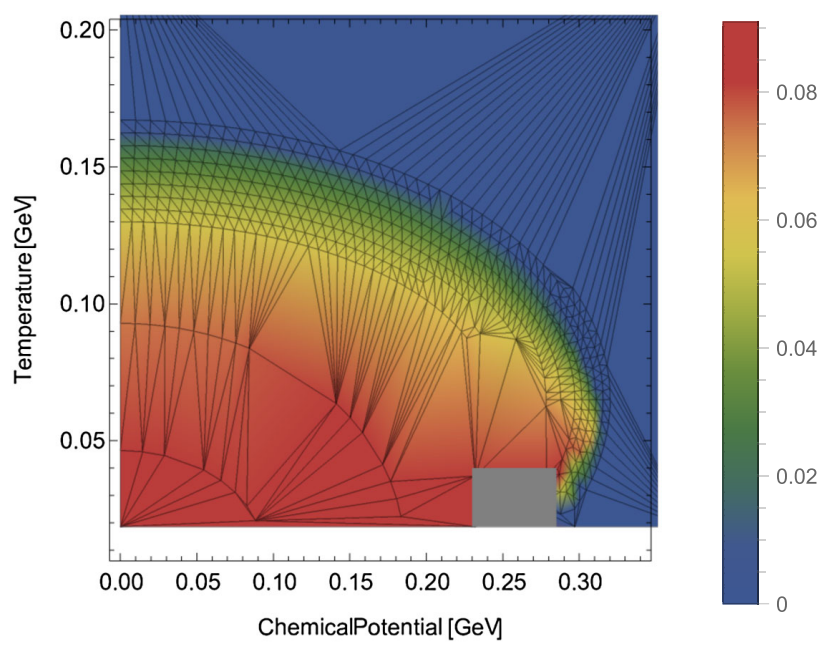

FIG. 10. Phase diagram for the quark-meson model in the large- $N$ limit with four d.o.f. with field-dependent Yukawa coupling. The gray box indicates the points that did not converge. The mesh indicates the discrete data points.

Appendix G. Importantly, apart from the flattening of the quark mass, they do not deviate significantly from the results in LPA. In particular this applies to their values of the equations of motion. Note, however, that this is bound to change for finite $N_{f}$.

\section{Phase structure}

As in LPA with a constant Yukawa coupling, we finally present our results on the phase diagram of the QM model in the large- $N_{f}$ limit including quark-meson scatterings via a field-dependent quark mass or Yukawa coupling.

The computation uses the same resolution as above: $N_{p}=2$ and $K=80$ cells in $\rho \in[0,0.02]$ up to the RG time $t=4$, that is, $k=0.001 \mathrm{GeV}$. The result is shown in Fig. 10. The computations did not complete the time integration for $\mu \geq 0.3 \mathrm{GeV}$; respective upgrades are currently being investigated.

As already discussed in the last section, Sec. IV C 1, in the phase with chiral symmetry breaking the fielddependent quark mass $m_{q}^{2}$ is necessarily flattened for $\rho \leq \rho_{0}$. In turn, the quark mass function does not flatten in the symmetric phase, and the quark mass is found to be zero in the symmetric phase. Plots of the field-dependent quark mass $m_{q}^{2}$ and pion mass $m_{\pi}^{2}$ for high values of temperature and density can be found in Appendix G and do not significantly deviate from the results with a constant Yukawa coupling.

We close this section with a comparison of the phase structure in Fig. 10 with that in LPA [Fig. 7(b)] in the same setting: large- $N_{f}$ limit with four d.o.f. While the phase boundaries do not change significantly, the crossover gets softened, if quark-meson scatterings are taken into account. This is clearly visible in Fig. 11, where we depict the chiral

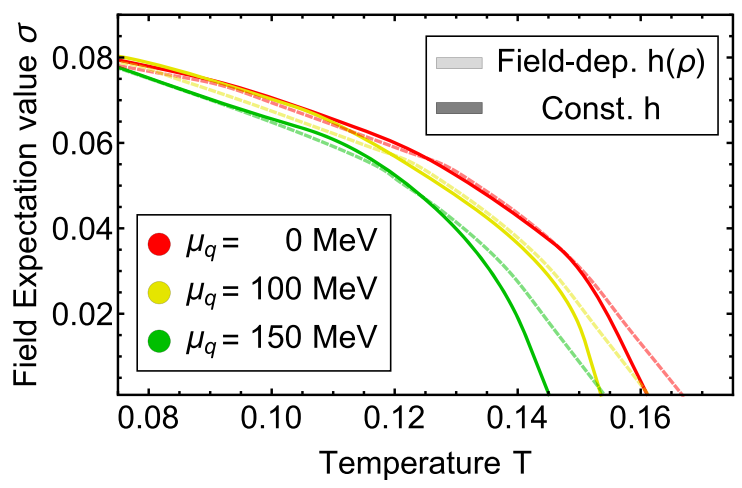

FIG. 11. Chiral order parameter $\sigma_{0}(T)$ in the large- $N_{f}$ limit with four d.o.f. as a function of temperature and quark chemical potential. We compare the LPA results with quark-meson scatterings encoded in $m_{q}(\sigma)$ for different densities. The data are interpolated from the phase diagrams in Figs. 7 and 10.

order parameter as a function of temperature for different densities with $\mu_{q}=0,100,150 \mathrm{MeV}$. This entails that the quark-meson scatterings considered here give sizable contributions to important observables measured in heavy-ion collisions. First of all, fluctuation observables will be sensitive to such a widening of the crossover. These effects may be even more prominent at large chemical potential where the freeze-out line most probably deviates from the chiral crossover line. Moreover, it can also be deduced from Fig. 11 that the quark-meson scatterings may have a sizable delaying effect on a possible critical end point. We conclude that these scatterings have to be taken into account for a quantitative prediction for the location of the CEP.

\section{CONCLUSIONS}

We have presented a study of the QM model with an emphasis on a quantitative access to order parameter potentials at finite chemical potentials. This allows us to discuss the eminently important question of the location of phase transition lines, that of the symmetry-breaking pattern and the order of the phase transitions.

The present study combines two systematic advances in the past years: The first one was the development of selfconsistent approximations for the computation of order parameter potentials [47]. The second one was the development of a numerical approach for solving flow equations that also enables us to discuss discontinuities in the flows such as shocks that are potentially relevant for the correct description of first- and second-order phase transitions [67].

Within this approach we have computed the phase structure of the QM model at finite temperature and density. An important benchmark is already provided in the large$N_{f}$ limit with an infinite number of flavors. We have argued that within an 't Hooft-type limit we can mimic the twoflavor QM model well (or any other flavor) and in particular 
reproduce well its nonuniversal properties such as the location of the phase boundary.

Moreover, in this limit the numerical approach within the discontinuous Galerkin setup in [67] is fully developed and we have a quantitative access to the shock development and propagation, even in the presence of nonconservative forces.

The present approach works very well except for a small regime at low temperature and onset densities. This is a merely technical problem and related upgrades of the present schemes are in development. Moreover, already for smaller ratios $\mu_{q} / T$ close to the crossover line we have to also improve the current approximation of the matter sector of QCD. This follows already from $[12,14]$. The results there indicate the potential relevance of nontrivial meson dispersions as well as the diquark channel at larger chemical potentials, $\mu_{q} / T \gtrsim 4 / 3$. Moreover, in the vicinity of a potential critical end point we also have to take into account the density channel, that mixed with the critical $\sigma$ mode. This is work in progress and we hope to report on it in the near future.

\section{ACKNOWLEDGMENTS}

We thank L. Corell, L. Kades, A. Koenigstein, F. Rennecke, M. Steil, and J. Urban for discussions. This work is done within the fQCD Collaboration [114], is supported by EMMI, the BMBF Grant No. 05P18VHFCA, and is part of and supported by the DFG Collaborative Research Centre SFB 1225 (ISOQUANT) as well as by the DFG under Germany's Excellence Strategy EXC-2181/1-390900948 (the Heidelberg Excellence Cluster STRUCTURES).

\section{APPENDIX A: IMPLEMENTATION OF DG METHODS IN DUNE}

This section gives an introduction to the numerical and computational framework used to solve Eq. (16). We made use of the Distributed and Unified Numerics Environment (DUNE) library [115-120], which is a modular toolbox for solving partial differential equations with grid-based methods. The library is an open source initiative to create a common interface for many different numerical methods and supports high-performance computing.

\section{Weak formulation and discrete problem}

The system of equations is solved on a computational domain $\Omega_{h}$, which is composed of $K$ disjoint elements, called cells $D^{k}$ such that

$$
\Omega \simeq \Omega_{h}=\bigcup_{k=1}^{K} D^{k} .
$$

For the purpose of the calculations in this work we used the DUNE grid YaspGrid, which is contained in the module dune-grid and allows for $n$-dimensional cubic grids and parallelized computation. In this paper a one-dimensional grid is used. It represents the computational domain $\Omega_{h}$, with the grid cells being disjoint intervals $D^{k}$ of possibly differing lengths, as discussed in Sec. III. In a more general formulation the domain $\Omega_{h}$ would be given as an $n$-dimensional rectangular grid and the elements $D^{k}$ would be implemented as cubic grid cells.

The solution in each cell $D^{k}$ is approximated by

$$
u(t, x) \simeq u_{h}(t, x)=\bigoplus_{k=1}^{K} u_{h}^{k}(t, x)
$$

where $u_{h}^{k}(t, x)$ is the local solution in each cell and the index $h$ denotes the approximation. The local solution in turn is then approximated by a polynomial of degree $N=N_{p}-1$ such that in each element $D^{k}$

$$
u_{h}^{k}(t, x)=\sum_{n=1}^{N_{p}} \hat{u}_{n}^{k}(t) q_{n}(x) .
$$

The local approximation $u_{h}^{k}(t, x)$ is given by a modal expansion, where $\left\{q_{n}\right\}$ is a local polynomial basis with time-dependent expansion coefficients $\hat{u}_{n}^{k}(t)$. Thus the global solution consists of $K$ local polynomial solutions of order $N$. The local approximation was implemented using the dune-pdelab module, specifically using the class QkDGLocalFiniteElementMap. In the one-dimensional case basis functions $q_{n}$ are given by the Legendre polynomials up to order $N_{p}$. For the purpose of higher-dimensional computations the basis functions are taken from the polynomial space $Q_{k}$ of the Legendre polynomials.

In our calculations we use a locally defined weak formulation of the convergence requirement:

$$
\begin{gathered}
\int_{D^{k}}\left(\left(\partial_{t} u_{i, h}+a_{i, h} \partial_{\rho} u_{i, h}+s_{i, h}\right) q_{n}+f_{i, h} \partial_{x} q_{n}\right) d x \\
=-\int_{\partial D^{k}} q_{n}\left(f_{i}^{*} \hat{\mathbf{n}}+\mathbf{D}\left(u_{i, h}^{+}, u_{i, h}^{-}, \hat{\mathbf{n}}\right)\right) d x .
\end{gathered}
$$

The right-hand side of the equation contains the standard numerical flux $f^{*}$ as well as an additional nonconservative flux term D. $\hat{\mathbf{n}}$ is the outward-pointing normal vector. We chose to use the local Lax-Friedrichs flux for $f^{*}$, which averages the flux on both sides of boundary and adds an additional diffusion term smoothing out jumps across the boundary:

$$
f^{*}\left(u_{h}^{+}, u_{h}^{-}\right)=\frac{1}{2}\left(f_{h}\left(u_{h}^{+}\right)+f_{h}\left(u_{h}^{-}\right)\right)+\frac{C}{2}\left[\left[\mathbf{u}_{\mathbf{h}}\right]\right],
$$

where the indices + and - denote the outward (neighboring) and interior element, respectively, at the boundary. The brackets denote a jump across the boundary: 


$$
[[\mathbf{u}]]=\left(\hat{\mathbf{n}}^{-} u^{-}+\hat{\mathbf{n}}^{+} u^{+}\right) .
$$

$f_{\max }$ is the local maximal wave speed, which corresponds to the speed of the fastest propagating mode across the boundary. In one dimension this corresponds to

$$
f_{\max } \geq \max _{D^{\{i, i+1\}}}\left|\partial_{u} f(u)\right| .
$$

The local Lax-Friedrichs flux is the most natural extension from the analytic solution of linear conservation laws to the nonlinear case. It relies on the so-called Roe condition, which reflects the assumption that the system is dominated by one strong wave.

\section{Nonconservative product}

The additional nonconservative flux across a boundary is given by $\mathbf{D}$. The theory of nonconservative fluxes was developed in $[80,81]$ and is applied in the context of finite volume and discontinuous Galerkin schemes [82-89,98]. To compute this quantity we need to consider the general form of a flux across an interface. For this purpose we consider a path $\phi_{i}(s)$ along the solution $u_{i}$, with start and end point $u_{i}^{L}$ and $u_{i}^{R}$, respectively, and the parameter $s \in[0,1]$. The formal definition of the flux along this path for a nonconservative flux contribution $a_{i} \partial_{\rho} u_{j}$ [see (16)] is then given by

$$
f_{i, \mathrm{nc}}=\int_{0}^{1} a_{i}\left(\phi_{i}(s), \phi_{j}(s), s\right) \partial_{s} \phi_{j}(s) d s .
$$

We remark that in the nonconservative case the flux is dependent on the chosen path.

By choosing the right and left sides of a boundary $u^{R}=$ $u^{+}$and $u^{L}=u^{-}$we are able to compute the flux from one cell to another. Similar to the numerical flux, D has to satisfy the jump property for consistency:

$$
\mathbf{D}\left(u^{+}, u^{-}, \hat{\mathbf{n}}\right)+\mathbf{D}\left(u^{+}, u^{-},-\hat{\mathbf{n}}\right)=\int_{0}^{1} a(\phi(s)) \hat{\mathbf{n}} \frac{\partial \phi}{\partial s} d s,
$$

which implies $D(u, u, \hat{\mathbf{n}})=0$ when there is no jump.

This condition can be obtained by integrating the equation around a discontinuity. It generalizes the so-called Rankine-Hugoniot condition for nonconservative systems of equations. The numerical fluxes are

$$
\begin{aligned}
\mathbf{D}\left(u^{+}, u^{-}, \hat{\mathbf{n}}\right)= & \frac{1}{2} \int_{0}^{1} a(\phi(s)) \hat{\mathbf{n}} \frac{\partial \phi}{\partial s} d s \\
& +\frac{1}{2} \int_{0}^{1}|a(\phi(s)) \hat{\mathbf{n}}| \frac{\partial \phi}{\partial s} d s .
\end{aligned}
$$

$|a(\phi(s)) \hat{\mathbf{n}}|$ is intended as the absolute value of the matrix namely $|a|=U^{-1} \operatorname{diag}\left(\left|\lambda_{1}\right|, \ldots,\left|\lambda_{N}\right|\right) U$, with $\lambda_{i}$ the eigenvalue of the matrix. It is possible to prove that this choice of flux reduces to the Lax-Friedrichs flux in the conservative case. If the dominant convection part of the equation is given by the conservative flux, this extra term can be neglected. The nonconservative flux under this assumption can therefore be computed from

$$
\mathbf{D}\left(u^{+}, u^{-}, \hat{\mathbf{n}}\right)=\frac{1}{2} \int_{0}^{1} a(\phi(s)) \hat{\mathbf{n}} \frac{\partial \phi}{\partial s} d s .
$$

The last remaining degree of freedom are the boundary conditions for the outer boundary of $\Omega_{h}$. In our case they are given by the in- or out-flowing flux, which is implemented by setting $u_{i, h}^{+}=u_{i, h}^{-}$at the outer boundaries, effectively adding an imaginary additional cell. It follows that the nonconservative flux is not fit for flux-boundary conditions, since the nonconservative flux vanishes at the outer boundaries due to the jump property. Therefore the equations need to be reformulated such that the boundary conditions can be met using the conservative flux. This is done in Appendix E.

\section{Time stepping}

The solution is computed by an explicit third-order timestepping scheme from the dune-pdelab module, where we additionally implemented Courant-Friedrichs-Lewy (CFL) conditions. The time step $\Delta t$ is thus limited by the propagation speed of the flow:

$$
\Delta t \leq \frac{\Delta x}{(2 N+1)} \frac{1}{f_{\max }},
$$

where $\Delta x$ is the size of the grid cell and $N$ the polynomial degree used in the computation, such that the denominator indicates the total amount of grid points within the respective cell. $f_{\max }$ is the maximal propagation speed of the information and was defined in (A4).

Additionally we use a minmod slope limiter before each computation step to suppress oscillations around kinks and jumps in the solution.

\section{APPENDIX B: CONVERGENCE PROPERTIES}

Results from the large- $N$ computations with constant Yukawa coupling (Sec. IV B) of different approximation orders $N_{p}$ are compared to benchmark the accuracy of the computations. Since there is no analytic solution available to compute the numerical error generated by the DG scheme, we use the result of a computation with $N_{p}=5$ and $K=700$ elements $u_{\text {ref }}$ as a reference. The results are compared at an energy scale of $k=140 \mathrm{MeV}$. The discrete time stepping was adjusted such that the time step computed by the CFL condition is lowered to ensure $k=$ $140 \mathrm{MeV}$ is reached exactly. The discrete solution is used to generate an interpolating function $u$, from which 


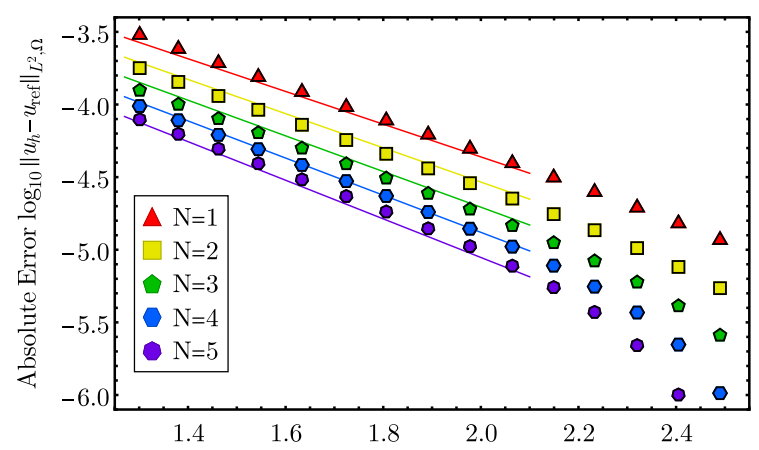

(a)

Number of Elements $\log _{10}(\mathrm{~K})$

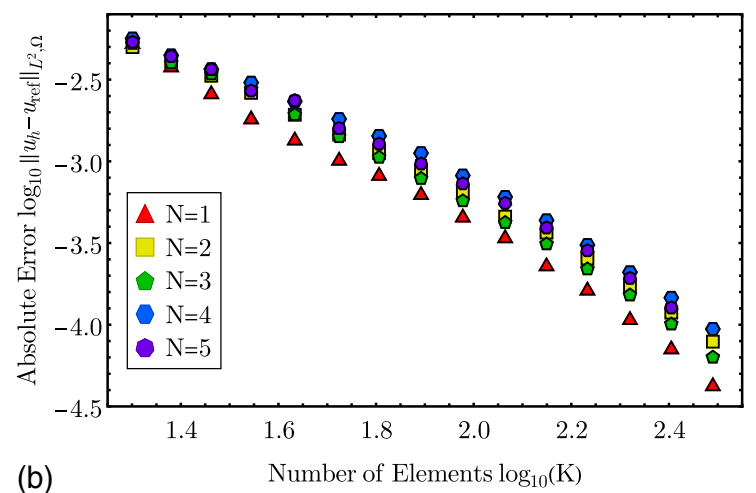

(b)

FIG. 12. Error of the solution with respect to the reference computation $u_{\text {ref }}$ with $K=700$ and $N_{p}=5$ at $T=10 \mathrm{MeV}$ and different values of the chemical potential. The solution was computed up to $k=150 \mathrm{MeV}$ in an interval $0 \leq \rho \leq 0.02$ for different numbers of equally sized cells $K$ and local approximation order $N_{p}$. The symbols show the result of the numerical simulation. (a) Error of the solution at $\mu=0 \mathrm{MeV}$. The lines show the $\chi^{2}$-fit of Eq. (B2) to all but the last 5 datapoints, with paramters given in Table V. (b) Error of the solution at $\mu=400 \mathrm{MeV}$.

the $L^{2}$ norm $\left\|u-u_{\text {ref }}\right\|_{L^{2}, \Omega_{h}}$ is computed. This is done at temperature $T=10 \mathrm{MeV}$ for $\mu=0 \mathrm{MeV}$ and $\mu=$ $400 \mathrm{MeV}$. The results are depicted in Figs. 12(a) and 12(b), respectively. In vacuum we recover good convergence properties observed in [67] and we perform a fit of the first ten data points to the parametrization:

$$
\begin{aligned}
& \log _{10}\left\|u-u_{\text {ref }}\right\|_{L^{2}, \Omega_{h}} \\
& =\left(a_{1}+a_{2} N_{p}\right)-\left(b_{1}+b_{2} N_{p}\right) \log _{10}(K) .
\end{aligned}
$$

The convergence behaves like a power law when increasing the number of elements $K$ and shows exponential convergence when increasing the local approximation order $N_{p}$ up to about $K=10^{2.1} \approx 125$. The fit parameters are given in Table $\mathrm{V}$ and the fit to the data points is included in Fig. 12(a). For higher $K$ the error decreases even faster which is due to the numerical error of $u_{\text {ref }}$ which e.g., also contains some of the resolution issues around the kink in the potential. When compared to [67] the relative error between $u$ and the reference solution $u_{\text {ref }}$ is significantly bigger. This is in part caused by the fact that the functions were interpolated and not reconstructed using the original basis polynomials, since the DUNE output only contains the values at cell boundaries with a precision of eight digits.

Adding a finite chemical potential increases the effect of the source term in Eq. (D1). At low temperatures the

TABLE V. Parameters obtained from a $\chi^{2}$ fit to the convergence behavior in Fig. 12(b) using Eq. (B2).

\begin{tabular}{lcccc}
\hline \hline Parameter & $a_{1}$ & $a_{2}$ & $b_{1}$ & $b_{2}$ \\
\hline$\chi^{2}$ fit & $2.016(80)$ & $-0.067(25)$ & $1.088(45)$ & $0.054(14)$ \\
\hline \hline
\end{tabular}

Silver-Blaze property introduces a sharp onset of chemical potential that shows better convergence for lower and odd $N_{p}$. However, since the exact solution is not known it is difficult to judge which local approximation is the best choice as a reference. Figure 12(b) illustrates the convergence properties in the area in which the source term dominates the equation over the flow.

\section{Convergence in systems with diffusion}

In this section we will comment on the convergence of the equations in the finite- $N_{f}$ case.

(i) We retain convergence properties similar to the previous section in regions of the phase diagram where the sigma mode is not critical. This is supported by the observation that the finite- $N_{f}$ and the large- $N_{f}$ simulations behave very similarly [see e.g., Figs. 2 and 4(a)]. The Courant number is a factor $C$ chosen to ensure the inequality in (A9). In hydrodynamics $C=0.01$ is a common choice for diffusive systems, which is appropriate if the flow is not diffusion dominated.

(ii) In the diffusion-dominated scenario, the solutions are not convergent. Additionally the time stepping becomes effectively 0 . This is due to shock development in the solution, which creates a steep negative slope, i.e., a very high (divergent) diffusion flux contribution.

The lack of convergence is explained by the fact that approximate Riemann solvers are only applicable in convection-dominated systems. The convergence of diffusion-dominated flows can only be ensured using a new formulation of the fluxes, such as the local-DG methods [121]. 


\section{Convergence with a nonconservative flux}

In this section we will comment on the convergence of the system with nonconservative flux, i.e., the computations for field-dependent Yukawa coupling.

We can again distinguish two cases.

(i) The area of the phase diagram where the pion mass $m_{\pi}^{2}$ has a single minimum for the entirety of the flow.-In this case we retain similar convergence properties as in section Appendix B.

(ii) At high chemical potential $\partial_{\rho} m_{\pi}^{2}$ begins to take on high negative values. - This directly feeds back to the nonconservative flux. In this area the nonconservative flux dominates the flow, which is not contained in the CFL conditions.

\section{APPENDIX C: CONVEXITY RESTORATION AND TIME STEPPING}

In this section we are going to inspect the time stepping and its related problems more closely. The equations are solved by a numerical stepwise integration of the RG time using the CFL conditions introduced in Appendix A 3. The size of the integration step is dependent on the information flux between cells, the local wave speed $f_{\max }$, which is defined in (A4). The local wave speed is plotted in Fig. 13 for approximate vacuum in the broken symmetry phase and for high temperatures and chemical potential in the symmetric phase.

\section{Approaching convexity in the broken symmetry phase}

It can be observed from Fig. 13 that the broken symmetry phase has a steadily increasing maximum wave speed. This corresponds to steadily decreasing time steps and leads to long computation times. This behavior is caused by the time-step inverse proportionality to the flux:

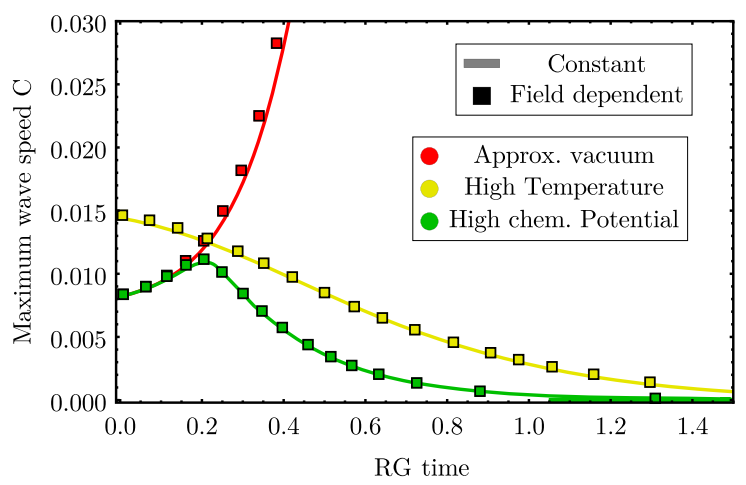

FIG. 13. Comparison of the maximal information propagation speed $f_{\max }$ for constant and field-dependent Yukawa couplings at different places in the phase diagram. Both computations were performed with $K=100$ cells and a local approximation order $N_{p}=2$.

$$
\Delta t \propto\left(k^{2}+u\right)^{n / 2},
$$

for some positive integer value of $n$. The two-point function $\Gamma^{(2)}$ can have negative eigenvalues during the RG flow, which is what happens to the computed function $u=m_{\pi}^{2}=\Gamma_{\pi \pi}^{(2)}$, the pion mass, in approximate vacuum. The flow is self-regulating, ensuring that the expression in the square root in the proportionality remains positive: The closer the root gets to becoming negative, the stronger the flow increases $u$, causing the modulo $|u|$ to always be slightly smaller than $k^{2}$. This must hold for $k \rightarrow 0$ from which it follows that $u \rightarrow 0$, such that $\Gamma^{(2)}=0$ at infinite RG time and convexity is restored. $|u|$ teeters on the edge of becoming bigger than $k$ during the entire integration which results in a big flux between grid cells and very small time steps.

\section{Convexity in the symmetric phase}

The pion mass becomes positive at some point during the interpolation in the symmetric phase and convexity is restored before $k=0$. Positive values of $u$ also significantly decrease the flux between grid cells as can be seen from Fig. 13 and increase the size of time steps.

At high temperatures the positiveness of $u$ is caused by the fact that the quark contribution to the flow that initially decreased $u$ is much smaller due to the dampening by the Fermi-Dirac distribution. This translates into $k^{2}-u$ never being remotely close to $|0|$ and therefore no increased convexity ensuring flux. Initially the maximum wave speed at high chemical potentials is similar to the approximate vacuum. However, the sudden onset of density at $\ln \left(\frac{\Gamma}{\mu}\right)$ drives $u$ to positive values and the information flux decreases.

It can be seen that the introduction of the field-dependent Yukawa coupling only slightly increases the wave speed in the broken symmetry phase and has no effect in the symmetric phase, which is to be expected from the observation that $m_{q}^{2}$ barely changes during the RG-time evolution at high temperatures or densities made in Sec. IV C.

\section{Problems and challenges with time stepping}

A recurrent struggle while solving the nonlinear RG equations using CFL conditions is time stepping. The RG flow continuously works to restore convexity, relying heavily on the fact that $k^{2}>|u|$ for negative $u$. For some cases where $k^{2}>|u|$ is very small, a numerical error, for example an oscillation around a shock, can cause the radicant to become negative and therefore no longer well defined. Limiting the time step by using (A4) is spoiled further at high densities, where the sigma mode becomes critical. The introduction of a steep slope in the potential introduces contributions to the flow that are not accounted for by time stepping. 


\section{APPENDIX D: FLOW EQUATIONS OF PION AND QUARK MASSES}

In this section the equations are reformulated to simplify their numerical treatment. The flow equation of the pion mass is obtained by taking a $\rho$ derivative of the effective potential. In case of the large- $N$ model this is given by

$$
\begin{aligned}
\partial_{t} u_{k}^{\mathrm{IN}}(\rho)= & \partial_{\rho}\left[\frac { k ^ { 5 } } { 1 2 \pi ^ { 2 } } \left\{\frac{N_{\pi}}{\epsilon_{k}^{\pi}}\left[1+2 n_{B}\left(\epsilon_{k}^{\pi}\right)\right]\right.\right. \\
& \left.\left.-\frac{4 \times 2 \times 3}{\epsilon_{k}^{q}}\left[1-n_{f}\left(\epsilon_{k}^{q}+\mu\right)-n_{f}\left(\epsilon_{k}^{q}-\mu\right)\right]\right\}\right] .
\end{aligned}
$$

The flow equation of the Yukawa coupling in (15) is rewritten in terms of the quark mass squared $m_{q}^{2}(\rho)$. To this aim, we multiply the original flow equation by $4 h(\rho) \rho$, which gives

$$
\begin{aligned}
\partial_{t} w_{k} & =4 h_{k} \rho A\left(u_{k}\right) \partial_{\rho} h_{k}+4 \rho h_{k}^{4} B\left(w_{k}, u_{k}\right) \\
& =2 \rho A\left(u_{k}\right) \partial_{\rho} h_{k}^{2}+4 \rho h_{k}^{4} B\left(w_{k}, u_{k}\right) \\
& =A\left(u_{k}\right) \partial_{\rho} w_{k}-2 h_{k}^{2} A\left(u_{k}\right)+4 \rho h_{k}^{4} B\left(w_{k}, u_{k}\right) \\
& =A\left(u_{k}\right) \partial_{\rho} w_{k}+\frac{w_{k}}{\rho}\left[w_{k} B\left(w_{k}, u_{k}\right)-A\left(u_{k}\right)\right],
\end{aligned}
$$

where

$$
A\left(m_{\pi, k}^{2} ; T, \mu\right)=-2 N_{\pi} v_{3} k^{2} l_{1}^{(B, 4)}\left(m_{\pi, k}^{2} ; T\right)
$$

corresponds to the contribution of the pion tadpole diagram and

$B\left(m_{q, k}^{2}, m_{\pi, k}^{2} ; T, \mu\right)=-4 N_{\pi} v_{3} L_{(1,1)}^{(4)}\left(m_{q, k}^{2}, m_{\pi, k}^{2} ; T, \mu\right)$

to the mixed contribution in Fig. 1. The explicit form of the threshold functions is given in Appendix $\mathrm{H}$.

\section{APPENDIX E: CALCULATION OF THE NONCONSERVATIVE NUMERICAL FLUX}

The flow equation for the Yukawa coupling (15) was reformulated in Appendix D to suit the general form of the partial differential equations given in (16) and contains a nonconservative flux term and a source term $s$ :

$$
\partial_{t} w_{k}=A\left(u_{k}\right) \partial_{\rho} w_{k}+s\left(u_{k}, w_{k}\right)
$$

The exact definition and derivation of the appearing terms are given in Appendix $\mathrm{H}$.

The nonconservative flux is computed using the integral derived by the jump condition in Appendix A in (A8). We chose a straight path across an interface:

$$
\begin{gathered}
w(s)=w^{-}+s\left(w^{+}-w^{-}\right), \\
u(s)=u^{-}+s\left(u^{+}-u^{-}\right) .
\end{gathered}
$$

We note again that this is a path along the solutions $u$ and $w$ and not a path in the "spatial" coordinate $\rho$. The straight path was chosen because it is often the simplest choice for the evaluation of the integral. In our case the expression simplifies so much that it can be evaluated analytically, due to the explicit form of the equations, where the nonconservative flux is given by

$$
A(u(s))=\partial_{u} g(u(s))=\frac{1}{u^{+}-u^{-}} \partial_{s} g(u(s)) .
$$

This gives

$$
\begin{aligned}
& \mathbf{D}\left(u^{+}, u^{-}, w^{+}, w^{-}, \hat{\mathbf{n}}\right) \\
& \quad=\frac{1}{2\left(u^{+}-u^{-}\right)} \int_{0}^{1} \hat{\mathbf{n}} \frac{\partial g(u(s))}{\partial s} \frac{\partial w(s)}{\partial s} d s+C[[\mathbf{w}]] \\
& \quad=\frac{\mathbf{n}}{2} \frac{g\left(u^{+}\right)-g\left(u^{-}\right)}{u^{+}-u^{-}}\left(w^{+}-w^{-}\right)+C[[\mathbf{w}]],
\end{aligned}
$$

where we used in the last equality that $\partial_{s} w$ is a constant expression. Instead, the constant $C$ is simply the absolute value of the Jacobian matrix:

$$
C=\int_{0}^{1}|A(u(s)) \hat{\mathbf{n}}| d s .
$$

Often, it can be approximated as the maximal characteristic speed of the nonconservative product. Note that for constant $u$ across the interface $\frac{g\left(u^{+}\right)-g\left(u^{-}\right)}{u^{+}-u^{-}}=A(u)$, such that we recover a conservative flux for constant $u$. There is a large set of paths across the interface that lead to the same value in the integral, due to the fact that $A$ can be written as a derivative of $u$. This hints at the possibility that there might be a conservative formulation for the system of equations.

Since this formulation only allows flux boundary conditions for conservative fluxes a partial integration is performed:

$$
A\left(u_{k}\right) \partial_{\rho} w_{k}=\partial_{\rho}\left(A\left(u_{k}\right) w_{k}\right)-w_{k} \partial_{\rho} A\left(u_{k}\right) .
$$

We now have a conservative flux $A\left(u_{k}\right) w_{k}$ the proper inand outflow boundary conditions for $w_{k}$ and a very small nonconservative flux contribution $\mathbf{D}^{\prime}$ accounting for jumps in $u_{k}$ :

$$
\mathbf{D}^{\prime}=\mathbf{D}-\left[A\left(u_{k}^{+}\right) w_{k}^{+}-A\left(u_{k}^{-}\right) w_{k}^{-}\right] .
$$

This contribution is very small when $u_{k}$ is smooth and only contains small jumps across interfaces. It obviously vanishes at the outer boundary since there we have $u_{k}^{+}=u_{k}^{-}$. Thanks to this formulation of the equation the maximal 

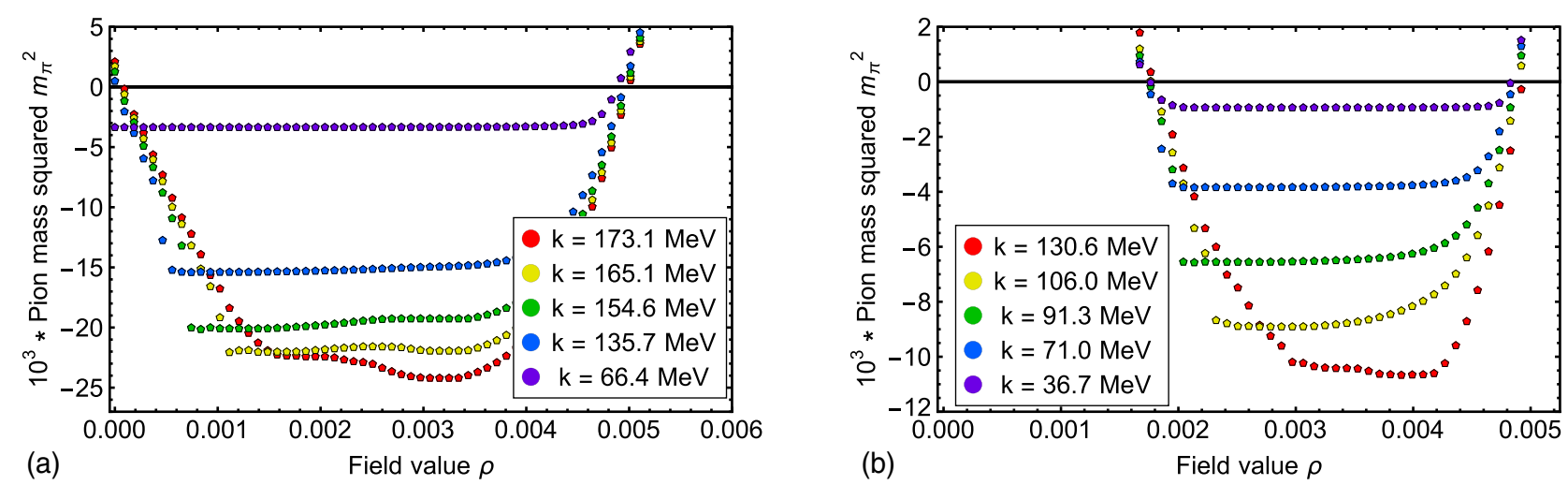

FIG. 14. Shock development at high densities. The potential derivative $\partial_{\rho} V_{k}(\rho)$ is plotted in the vicinity of the phase transition at $\mu=0.30 \mathrm{GeV}$ and $\mu=0.32 \mathrm{GeV}$ in the large- $N_{f}$ limit with $N_{\pi}=3$. The numerical oscillations around the shock were flattened out by a minmod slope limiter. The figure depicts the grid points on which the computation was carried out. (a) Derivative of the potential in the broken phase. The developing shock travels to unphysical values of the field value $\rho$ and the potential is flattened out. (b) Derivative of the potential in the symmetric phase. The developing shock freezes in at finite $\rho$, creating a global mimumum at $\rho=0$.

wave speed of the nonconservative product is rather small and can be neglected in practice. In the general case however the inclusion of this term is important especially if the nonconservative product is the only convective term in the equation, since it introduces the necessary numerical dissipation to make the numerical scheme stable.

\section{APPENDIX F: SHOCK DEVELOPMENT}

In this section we illustrate the dynamics of shock development in the large- $N$ model. In Sec. IV we distinguished two scenarios of shock development which are both illustrated in Fig. 14. Figure 14(a) shows the pion mass and the potential derivative in the broken symmetry phase, where the shock eventually proceeds to move to unphysical values for $\rho$. This leads to the creation of a temporary maximum in the potential, depicted in Fig. 15(a), which flattens out again for $k \rightarrow 0$. Figure 15(a) shows the typical form of a potential in the broken symmetry phase with a degenerate global minimum at $k=0$. In Fig. 14(b) the shock freezes at some positive finite value. The temporary maximum still vanishes due to convexity restoration; however, it remains at a positive value such that the potential in the symmetric phase has a unique global minimum at $\sigma_{0}=0 \mathrm{GeV}$. The shock position and the position of the temporary maximum coincide in the deep infrared.

\section{APPENDIX G: FIELD-DEPENDENT PION AND QUARK MASSES}

Here we provide plots for the field-dependent quark mass $m_{q}^{2}$ and pion mass $m_{\pi}^{2}$ for the case of high temperatures and high chemical potential in the symmetric phase. It can be seen from Figs. 16(a) and 16(b) that the RG flow generates massive pions, similar to the computations with constant Yukawa coupling. The quark mass is expected to vanish in the symmetric phase as can be seen from Fig. 16(d).
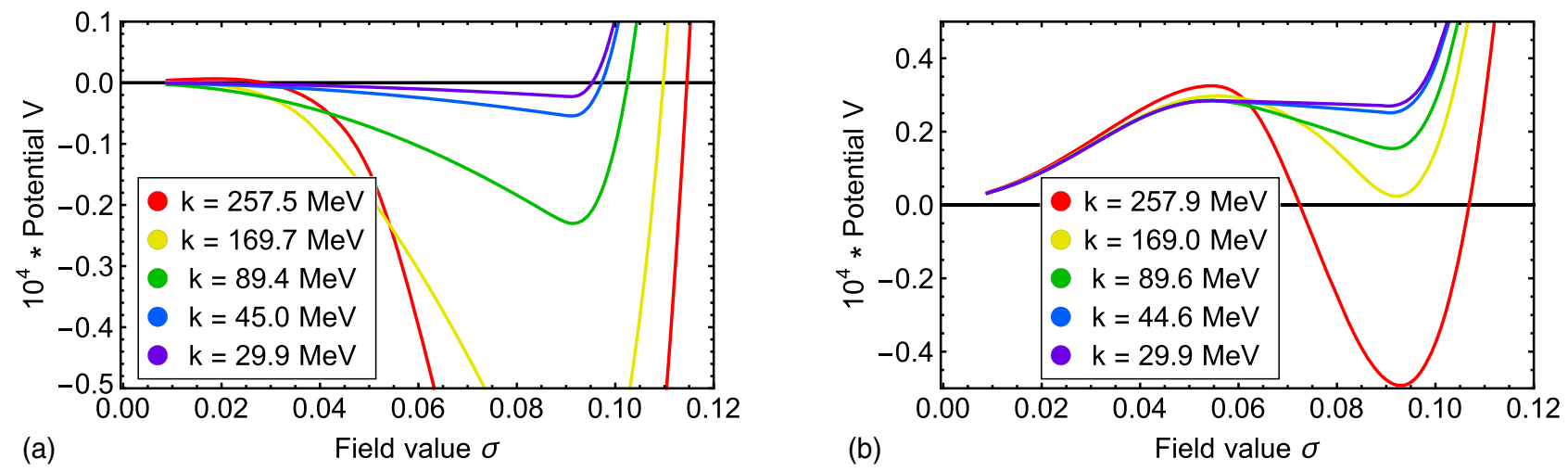

FIG. 15. The effective potential $V_{k}(\rho)$ in the vicinity of the phase transition at $\mu=0.30 \mathrm{GeV}$ and $\mu=0.32 \mathrm{GeV}$ in the large- $N_{f}$ limit with $N_{\pi}=3$. (a) Potential in the broken phase. (b) Potential in the symmetric phase. 

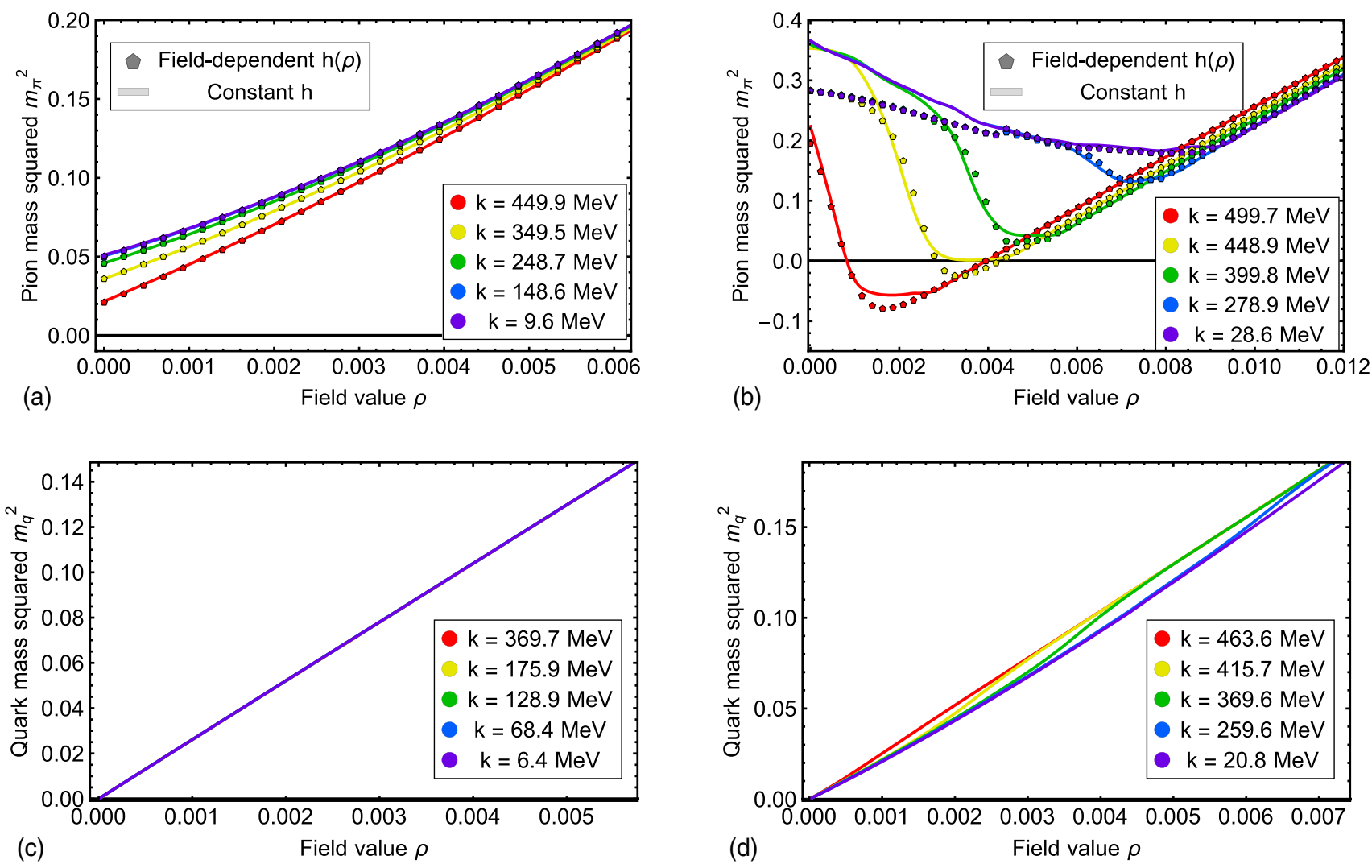

FIG. 16. RG-time evolution of the pion and quark masses in the broken symmetry phase. The computation was performed using $K=120$ cells and a local approximation order of $N_{p}=2$. (a) Pion mass at high temperatures ( $\left.T=280 \mathrm{MeV}\right)$ and zero density. (b) Pion mass at high densities $(\mu=460 \mathrm{MeV})$ and zero temperature. (c) Quark mass at high temperatures $(T=280 \mathrm{MeV})$ and zero density. (d) Quark mass at high densities $(\mu=460 \mathrm{MeV})$ and zero temperature.

\section{APPENDIX H: REGULATORS AND THRESHOLD FUNCTIONS}

In the present work we use the three-dimensional flat or Litim regulator; see [78]. The flat bosonic regulator is

$R_{\phi}(p)=p^{2} r_{\phi}(x), \quad r_{\phi}(x)=\left(\frac{1}{x}-1\right) \theta(1-x)$,

with $x=p^{2} / k^{2}$, and the fermionic one reads

$$
R_{q}=\not p r_{q}(x), \quad r_{q}(x)=\left(\frac{1}{\sqrt{x}}-1\right) \theta(1-x) .
$$

The threshold functions $l_{1}^{B / F, d}$ and $L_{n, m}^{(d)}$ in (D3) and (D4) are taken from [47] and shown for the sake of completeness for $Z_{\phi}=Z_{q}=1$; see (4).

The functions $l_{0}^{(B / F, d)}$ are deduced from bosonic and fermionic loops in $d$ dimensions, to wit,

$$
\begin{aligned}
l_{0}^{(B / F, d)}\left(\hat{m}_{\phi, k}^{2} ; T\right) & =\frac{T}{2 k} \sum_{n \in \mathbb{Z}} \int d x x^{\frac{d-1}{2}} \frac{\partial_{t} r_{\phi}(x)-r_{\phi}(x)}{\frac{\omega_{n}^{2}}{k^{2}}+x\left[1+r_{\phi}(x)\right]+m_{\phi, k}^{2}} \\
& =\frac{2}{d-1} \frac{1}{\sqrt{1+\hat{m}_{\phi, k}^{2}}}\left[\frac{1}{2}+n_{B}\left(T, \hat{m}_{\phi, k}^{2}\right)\right] .
\end{aligned}
$$

In (H3), $n_{B}$ is the Bose-Einstein distribution and $\hat{m}^{2}=$ $m^{2} / k^{2}$ the dimensionless masses. The threshold functions $l_{n}^{(B / F, d)}$ for diagrams with loops containing $n+1$ bosonic and fermionic propagator terms are obtained by taking a derivative with respect to $\hat{m}^{2}$ :

$$
\partial_{\hat{m}^{2}} l_{n}^{(B / F, d)}\left(\hat{m}^{2}\right)=-\left(n+\delta_{n 0}\right) l_{n+1}^{(B / F, d)}\left(\hat{m}^{2}\right) .
$$

Thus the function $l_{1}^{(B, 4)}$ corresponds to the last term in Fig. 1, containing a bosonic regulator and two propagators.

The function $L_{(1,1)}^{(4)}$ in (D4) consists of the first two terms in Fig. 1. It can be obtained with a $\hat{m}_{q, k}^{2}$ derivative from $\mathcal{F} \mathcal{B}_{(1,1)}$ that describes a loop with one fermionic and one bosonic propagator: 


$$
\begin{aligned}
& \mathcal{F B}_{(1,1)}\left(\hat{m}_{q, k}^{2}, \hat{m}_{\phi, k}^{2} ; T, \mu\right)=\frac{T}{k} \operatorname{Re}\left[\sum_{n \in \mathbb{Z}} \frac{1}{\frac{\omega_{n}^{2}}{k^{2}}+x\left[1+r_{\phi}(x)\right]+\hat{m}_{\phi, k}^{2}} \times \frac{1}{\frac{\left(\nu_{n}+i \mu\right)^{2}}{k^{2}}+x\left[1+r_{q}(x)\right]^{2}+m_{q, k}^{2}}\right] \\
& =\operatorname{Re}\left\{\frac{1}{2 \sqrt{1+\hat{m}_{\phi, k}^{2}}}\left[\frac{1}{2}+n_{B}\left(T, \hat{m}_{\phi, k}^{2}\right)\right]\right. \\
& \times\left[\frac{1}{\hat{m}_{q, k}^{2}+1-\left(\frac{\mu}{k}-\frac{i \pi T}{k}-\sqrt{1+\hat{m}_{\phi, k}^{2}}\right)^{2}}+\frac{1}{\hat{m}_{q, k}^{2}+1-\left(\frac{\mu}{k}-\frac{i \pi T}{k}+\sqrt{1+\hat{m}_{\phi, k}^{2}}\right)^{2}}\right] \\
& -\frac{1}{2 \sqrt{1+\hat{m}_{q, k}^{2}}}\left(n_{F}\left(T,-\mu, \hat{m}_{q, k}^{2}\right)-\frac{1}{2} \frac{1}{\hat{m}_{\phi, k}^{2}+1-\left(\frac{\mu}{k}-\frac{i \pi T}{k}-\sqrt{1+\hat{m}_{q, k}^{2}}\right)^{2}}\right) \\
& \left.-\frac{1}{2 \sqrt{1+\hat{m}_{q, k}^{2}}}\left(n_{F}\left(T, \mu, \hat{m}_{q, k}^{2}\right)-\frac{1}{2} \frac{1}{\hat{m}_{\phi, k}^{2}+1-\left(\frac{\mu}{k}-\frac{i \pi T}{k}+\sqrt{1+\hat{m}_{q, k}^{2}}\right)^{2}}\right)\right\},
\end{aligned}
$$

where

$$
n_{F}\left(T, \mu, \hat{m}_{q, k}^{2}\right)=\frac{1}{\exp \left\{\frac{k}{T}\left(\sqrt{1+\hat{m}_{q, k}^{2}}+\frac{\mu}{k}\right)\right\}+1}
$$

$L_{(1,1)}^{(4)}$ can now be generated by taking derivatives with respect to the fermionic mass $\hat{m}_{q, k}^{2}$ and the bosonic mass $\hat{m}_{\phi, k}^{2}$. These derivatives correspond to multiplying a fermionic or bosonic propagator to the loop in $\mathcal{F B}_{(1,1)}$ :

$$
\partial_{\hat{m}_{q, k}^{2}} \mathcal{F} \mathcal{B}_{(m, n)}=-m \mathcal{F} \mathcal{B}_{(m+1, n)}, \quad \partial_{\hat{m}_{\phi, k}^{2}} \mathcal{F} \mathcal{B}_{(m, n)}=-n \mathcal{F} \mathcal{B}_{(m, n+1)}
$$

This leads us to

$$
\begin{aligned}
L_{(1,1)}^{(d)}= & \frac{T}{2 k} \sum_{n \in \mathbb{Z}} \int d x x^{\frac{d-1}{2}}\left\{\frac{\partial_{t} r_{\phi}(x)}{\frac{\nu_{n}^{2}}{k^{2}}+x\left[1+r_{q}(x)\right]^{2}+m_{q, k}^{2}} \times\left(\frac{1}{\frac{\omega_{n}^{2}}{k^{2}}+x\left[1+r_{\phi}(x)\right]+m_{\phi, k}^{2}}\right)^{2}\right. \\
& \left.+\frac{2 r_{q}(x)}{\frac{\omega_{n}^{2}}{k^{2}}+x\left[1+r_{\phi}(x)\right]+m_{\phi, k}^{2}} \times\left(\frac{1}{\frac{\nu_{n}^{2}}{k^{2}}+x\left[1+r_{q}(x)\right]^{2}+m_{q, k}^{2}}\right)^{2}\right\} \\
= & \frac{2}{d-1}\left(\mathcal{F B}_{(1,2)}+\mathcal{F B}_{(2,1)}\right) .
\end{aligned}
$$

[1] X. Luo and N. Xu, Search for the QCD critical point with fluctuations of conserved quantities in relativistic heavyion collisions at RHIC: An overview, Nucl. Sci. Tech. 28, 112 (2017).

[2] L. Adamczyk et al. (STAR Collaboration), Bulk properties of the medium produced in relativistic heavy-ion collisions from the beam energy scan program, Phys. Rev. C 96, 044904 (2017).
[3] A. Andronic, P. Braun-Munzinger, K. Redlich, and J. Stachel, Decoding the phase structure of QCD via particle production at high energy, Nature (London) 561, 321 (2018).

[4] M. A. Stephanov, QCD phase diagram: An overview, Proc. Sci., LAT2006 (2006) 024.

[5] J. O. Andersen, W. R. Naylor, and A. Tranberg, Phase diagram of QCD in a magnetic field: A review, Rev. Mod. Phys. 88, 025001 (2016). 
[6] E. Shuryak, Strongly coupled quark-gluon plasma in heavy ion collisions, Rev. Mod. Phys. 89, 035001 (2017).

[7] J. M. Pawlowski, Equation of state and phase diagram of strongly interacting matter, Nucl. Phys. A931, 113 (2014).

[8] C. D. Roberts and S. M. Schmidt, Dyson-Schwinger equations: Density, temperature and continuum strong QCD, Prog. Part. Nucl. Phys. 45, S1 (2000).

[9] C.S. Fischer, QCD at finite temperature and chemical potential from Dyson-Schwinger equations, Prog. Part. Nucl. Phys. 105, 1 (2019).

[10] Y. Yin, The QCD critical point hunt: Emergent new ideas and new dynamics, arXiv:1811.06519.

[11] W.-J. Fu, J. M. Pawlowski, and F. Rennecke, Strangeness neutrality and QCD thermodynamics, SciPost Phys. Core 2, 2 (2020).

[12] W.-J. Fu, J. M. Pawlowski, and F. Rennecke, QCD phase structure at finite temperature and density, Phys. Rev. D 101, 054032 (2020).

[13] M. Leonhardt, M. Pospiech, B. Schallmo, J. Braun, C. Drischler, K. Hebeler, and A. Schwenk, Symmetric Nuclear Matter from the Strong Interaction, Phys. Rev. Lett. 125, 142502 (2020).

[14] J. Braun, M. Leonhardt, and M. Pospiech, Fierz-complete NJL model study III: Emergence from quark-gluon dynamics, Phys. Rev. D 101, 036004 (2020).

[15] J. Braun, W.-J. Fu, J. M. Pawlowski, F. Rennecke, D. Rosenblüh, and S. Yin, Chiral susceptibility in $(2+1)-$ flavor QCD, Phys. Rev. D 102, 056010 (2020).

[16] K. Otto, M. Oertel, and B.-J. Schaefer, Nonperturbative quark matter equations of state with vector interactions, Eur. Phys. J. ST 229, 3629 (2020).

[17] N. Dupuis, L. Canet, A. Eichhorn, W. Metzner, J. Pawlowski, M. Tissier, and N. Wschebor, The nonperturbative functional renormalization group and its applications, Phys. Rep. 910, 1 (2021).

[18] W.-J. Fu, X. Luo, J. M. Pawlowski, F. Rennecke, R. Wen, and S. Yin, Hyper-order baryon number fluctuations at finite temperature and density, arXiv:2101.06035.

[19] Y.-R. Chen, R. Wen, and W.-J. Fu, Critical behaviors of the $O(4)$ and $Z(2)$ symmetries in the QCD phase diagram, arXiv:2101.08484.

[20] C. S. Fischer, J. Luecker, and C. A. Welzbacher, Phase structure of three and four flavor QCD, Phys. Rev. D 90, 034022 (2014).

[21] F. Gao and Y.-X. Liu, QCD phase transitions via a refined truncation of Dyson-Schwinger equations, Phys. Rev. D 94, 076009 (2016).

[22] P. Isserstedt, M. Buballa, C. S. Fischer, and P. J. Gunkel, Baryon number fluctuations in the QCD phase diagram from Dyson-Schwinger equations, Phys. Rev. D 100, 074011 (2019).

[23] F. Gao and J.M. Pawlowski, QCD phase structure from functional methods, Phys. Rev. D 102, 034027 (2020).

[24] F. Gao and J. M. Pawlowski, Chiral phase structure and critical end point in QCD, arXiv:2010.13705.

[25] A. Bazavov et al., The QCD equation of state to $\mathcal{O}\left(\mu_{B}^{6}\right)$ from lattice QCD, Phys. Rev. D 95, 054504 (2017).
[26] A. Bazavov et al. (HotQCD Collaboration), Skewness and kurtosis of net baryon-number distributions at small values of the baryon chemical potential, Phys. Rev. D 96, 074510 (2017).

[27] C. Bonati, M. D’Elia, F. Negro, F. Sanfilippo, and K. Zambello, Curvature of the pseudocritical line in QCD: Taylor expansion matches analytic continuation, Phys. Rev. D 98, 054510 (2018).

[28] S. Borsanyi, Z. Fodor, J. N. Guenther, S. K. Katz, K. K. Szabo, A. Pasztor, I. Portillo, and C. Ratti, Higher order fluctuations and correlations of conserved charges from lattice QCD, J. High Energy Phys. 10 (2018) 205.

[29] A. Bazavov et al. (HotQCD Collaboration), Chiral crossover in QCD at zero and non-zero chemical potentials, Phys. Lett. B 795, 15 (2019).

[30] J. N. Guenther, S. Borsanyi, Z. Fodor, S. K. Katz, K. K. Szab, A. Pasztor, I. Portillo, and C. Ratti, Lattice thermodynamics at finite chemical potential from analytical continuation, J. Phys. Conf. Ser. 1070, 012002 (2018).

[31] H. Ding et al., Chiral Phase Transition Temperature in $(2+1)$-Flavor QCD, Phys. Rev. Lett. 123, 062002 (2019).

[32] S. Borsanyi, Z. Fodor, J. N. Guenther, R. Kara, S. D. Katz, P. Parotto, A. Pasztor, C. Ratti, and K. K. Szabo, The QCD Crossover at Finite Chemical Potential from Lattice Simulations, Phys. Rev. Lett. 125, 052001 (2020).

[33] H. Gies and C. Wetterich, Renormalization flow of bound states, Phys. Rev. D 65, 065001 (2002).

[34] H. Gies and C. Wetterich, Universality of spontaneous chiral symmetry breaking in gauge theories, Phys. Rev. D 69, 025001 (2004).

[35] J. M. Pawlowski, Aspects of the functional renormalisation group, Ann. Phys. (Amsterdam) 322, 2831 (2007).

[36] S. Floerchinger and C. Wetterich, Exact flow equation for composite operators, Phys. Lett. B 680, 371 (2009).

[37] V. Skokov, B. Stokic, B. Friman, and K. Redlich, Meson fluctuations and thermodynamics of the Polyakov loop extended quark-meson model, Phys. Rev. C 82, 015206 (2010).

[38] T. K. Herbst, J. M. Pawlowski, and B.-J. Schaefer, The phase structure of the Polyakovquarkmeson model beyond mean field, Phys. Lett. B 696, 58 (2011).

[39] K. Kamikado, T. Kunihiro, K. Morita, and A. Ohnishi, Functional renormalization group study of phonon mode effects on chiral critical point, Prog. Theor. Exp. Phys. 2013, 053D01 (2013).

[40] J. Braun and T. K. Herbst, On the relation of the deconfinement and the chiral phase transition in gauge theories with fundamental and adjoint matter, arXiv:1205.0779.

[41] R.-A. Tripolt, J. Braun, B. Klein, and B.-J. Schaefer, Effect of fluctuations on the QCD critical point in a finite volume, Phys. Rev. D 90, 054012 (2014).

[42] T. K. Herbst, J. M. Pawlowski, and B.-J. Schaefer, Phase structure and thermodynamics of QCD, Phys. Rev. D 88, 014007 (2013).

[43] T. K. Herbst, M. Mitter, J. M. Pawlowski, B.-J. Schaefer, and R. Stiele, Thermodynamics of QCD at vanishing density, Phys. Lett. B 731, 248 (2014).

[44] M. Mitter and B.-J. Schaefer, Fluctuations and the axial anomaly with three quark flavors, Phys. Rev. D 89, 054027 (2014). 
[45] M. Drews, T. Hell, B. Klein, and W. Weise, Thermodynamic phases and mesonic fluctuations in a chiral nucleonmeson model, Phys. Rev. D 88, 096011 (2013).

[46] A. J. Helmboldt, J. M. Pawlowski, and N. Strodthoff, Towards quantitative precision in the chiral crossover: Masses and fluctuation scales, Phys. Rev. D 91, 054010 (2015).

[47] J. M. Pawlowski and F. Rennecke, Higher order quarkmesonic scattering processes and the phase structure of QCD, Phys. Rev. D 90, 076002 (2014).

[48] W.-J. Fu and J. M. Pawlowski, Relevance of matter and glue dynamics for baryon number fluctuations, Phys. Rev. D 92, 116006 (2015).

[49] Y. Jiang, T. Xia, and P. Zhuang, Topological susceptibility in three-flavor quark meson model at finite temperature, Phys. Rev. D 93, 074006 (2016).

[50] W.-J. Fu, J. M. Pawlowski, F. Rennecke, and B.-J. Schaefer, Baryon number fluctuations at finite temperature and density, Phys. Rev. D 94, 116020 (2016).

[51] F. Rennecke and B.-J. Schaefer, Fluctuation-induced modifications of the phase structure in $(2+1)$-flavor QCD, Phys. Rev. D 96, 016009 (2017).

[52] H. Zhang, D. Hou, T. Kojo, and B. Qin, Functional renormalization group study of the quark-meson model with $\omega$ meson, Phys. Rev. D 96, 114029 (2017).

[53] R.-A. Tripolt, B.-J. Schaefer, L. von Smekal, and J. Wambach, Low-temperature behavior of the quark-meson model, Phys. Rev. D 97, 034022 (2018).

[54] S. Resch, F. Rennecke, and B.-J. Schaefer, Mass sensitivity of the three-flavor chiral phase transition, Phys. Rev. D 99, 076005 (2019).

[55] W.-J. Fu, Chiral criticality and glue dynamics, Chin. Phys. C 43, 074101 (2019).

[56] W.-J. Fu, J. M. Pawlowski, and F. Rennecke, Strangeness neutrality and baryon-strangeness correlations, Phys. Rev. D 100, 111501 (2019).

[57] S. Yin, R. Wen, and W.-J. Fu, Mesonic dynamics and QCD phase transition, Phys. Rev. D 100, 094029 (2019).

[58] R. Wen and W.-J. Fu, Correlations of conserved charges and QCD phase structure, Chin. Phys. C 45, 044112 (2021).

[59] R. C. Pereira, R. Stiele, and P. Costa, Functional renormalization group study of the critical region of the quarkmeson model with vector interactions, Eur. Phys. J. C 80, 712 (2020).

[60] J. Braun, T. Dörnfeld, B. Schallmo, and S. Töpfel, Renormalization group studies of dense relativistic systems, arXiv:2008.05978.

[61] A. Connelly, G. Johnson, F. Rennecke, and V. Skokov, Universal Location of the Yang-Lee Edge Singularity in $O(N)$ Theories, Phys. Rev. Lett. 125, 191602 (2020).

[62] J. Braun, The QCD phase boundary from quark-gluon dynamics, Eur. Phys. J. C 64, 459 (2009).

[63] J. Braun, L. Fister, J. M. Pawlowski, and F. Rennecke, From quarks and gluons to hadrons: Chiral symmetry breaking in dynamical QCD, Phys. Rev. D 94, 034016 (2016).

[64] M. Mitter, J. M. Pawlowski, and N. Strodthoff, Chiral symmetry breaking in continuum QCD, Phys. Rev. D 91, 054035 (2015).
[65] A. K. Cyrol, M. Mitter, J. M. Pawlowski, and N. Strodthoff, Nonperturbative quark, gluon, and meson correlators of unquenched QCD, Phys. Rev. D 97, 054006 (2018).

[66] R. Alkofer, A. Maas, W. A. Mian, M. Mitter, J. París-López, J. M. Pawlowski, and N. Wink, Bound state properties from the functional renormalization group, Phys. Rev. D 99, 054029 (2019).

[67] E. Grossi and N. Wink, Resolving phase transitions with discontinuous Galerkin methods, arXiv:1903.09503.

[68] F. Rennecke, Vacuum structure of vector mesons in QCD, Phys. Rev. D 92, 076012 (2015).

[69] J. Berges, N. Tetradis, and C. Wetterich, Nonperturbative renormalization flow in quantum field theory and statistical physics, Phys. Rep. 363, 223 (2002).

[70] B.-J. Schaefer and J. Wambach, Renormalization group approach towards the QCD phase diagram, Phys. Part. Nucl. 39, 1025 (2008).

[71] H. Gies, Introduction to the functional RG and applications to gauge theories, Lect. Notes Phys. 852, 287 (2012).

[72] J. Braun, Fermion interactions and universal behavior in strongly interacting theories, J. Phys. G 39, 033001 (2012).

[73] B.-J. Schaefer and J. Wambach, The phase diagram of the quark meson model, Nucl. Phys. A757, 479 (2005).

[74] G. P. Vacca and L. Zambelli, Multimeson Yukawa interactions at criticality, Phys. Rev. D 91, 125003 (2015).

[75] A. Jakovac, I. Kaposvari, and A. Patkos, Scalar mass stability bound in a simple Yukawa-theory from renormalization group equations, Mod. Phys. Lett. A 32, 1750011 (2017).

[76] H. Gies, R. Sondenheimer, and M. Warschinke, Impact of generalized Yukawa interactions on the lower Higgs mass bound, Eur. Phys. J. C 77, 743 (2017).

[77] G. Fejos and A. Patkos, Field dependence of the Yukawa coupling in the three flavor quark-meson model, Phys. Rev. D 103, 056015 (2021).

[78] D. F. Litim, Critical exponents from optimized renormalization group flows, Nucl. Phys. B631, 128 (2002).

[79] L. Rezzolla and O. Zanotti, Relativistic Hydrodynamics (Oxford Scholarship Online, 2014), https://doi.org/ 10.1093/acprof:oso/9780198528906.001.0001.

[80] C. Parés, Numerical methods for nonconservative hyperbolic systems: A theoretical framework, SIAM J. Numer. Anal. 44, 300 (2006).

[81] M. Castro, J. Gallardo, and C. Parés, High order finite volume schemes based on reconstruction of states for solving hyperbolic systems with nonconservative products. Applications to shallow-water systems, Math. Comput. 75, 1103 (2006).

[82] T. Y. Hou and P. G. LeFloch, Why nonconservative schemes converge to wrong solutions: Error analysis, Math. Comput. 62, 497 (1994).

[83] M. J. Castro, J. M. Gallardo, and A. Marquina, Approximate osher-solomon schemes for hyperbolic systems, Appl. Math. Comput. 272, 347 (2016), recent Advances in numerical methods for hyperbolic partial differential equations. 
[84] M. Dumbser, A. Hidalgo, M. Castro, C. Parés, and E. F. Toro, Force schemes on unstructured meshes II: Non-conservative hyperbolic systems, Comput. Methods Appl. Mech. Eng. 199, 625 (2010).

[85] M. Dumbser, M. Castro, C. Parés, and E. F. Toro, Ader schemes on unstructured meshes for nonconservative hyperbolic systems: Applications to geophysical flows, Comput. Fluids 38, 1731 (2009).

[86] M. Castro, A. Pardo, C. Parés, and E. Toro, On some fast well-balanced first order solvers for nonconservative systems, Math. Comput. 79, 1427 (2010).

[87] M. J. Castro, P. G. LeFloch, M. L. Muñoz-Ruiz, and C. Parés, Why many theories of shock waves are necessary: Convergence error in formally path-consistent schemes, J. Comput. Phys. 227, 8107 (2008).

[88] M. Dumbser and E. F. Toro, A simple extension of the osher riemann solver to non-conservative hyperbolic systems, J. Sci. Comput. 48, 70 (2011).

[89] Discontinuous galerkin finite element methods for hyperbolic nonconservative partial differential equations, J. Comput. Phys. 227, 1887 (2008).

[90] P. Bozek, Flow and interferometry in $3+1$ dimensional viscous hydrodynamics, Phys. Rev. C 85, 034901 (2012).

[91] B. Schenke, S. Jeon, and C. Gale, $(3+1)$ D hydrodynamic simulation of relativistic heavy-ion collisions, Phys. Rev. C 82, 014903 (2010).

[92] I. Karpenko, P. Huovinen, and M. Bleicher, A 3+1 dimensional viscous hydrodynamic code for relativistic heavy ion collisions, Comput. Phys. Commun. 185, 3016 (2014).

[93] C. Shen, Z. Qiu, H. Song, J. Bernhard, S. Bass, and U. Heinz, The iEBE-VISHNU code package for relativistic heavy-ion collisions, Comput. Phys. Commun. 199, 61 (2016).

[94] L. Del Zanna, V. Chandra, G. Inghirami, V. Rolando, A. Beraudo, A. De Pace, G. Pagliara, A. Drago, and F. Becattini, Relativistic viscous hydrodynamics for heavyion collisions with ECHO-QGP, Eur. Phys. J. C 73, 2524 (2013).

[95] J. Noronha-Hostler, G. S. Denicol, J. Noronha, R. P. G. Andrade, and F. Grassi, Bulk viscosity effects in event-byevent relativistic hydrodynamics, Phys. Rev. C 88, 044916 (2013).

[96] S. Floerchinger and E. Grossi, Causality of fluid dynamics for high-energy nuclear collisions, J. High Energy Phys. 08 (2018) 186.

[97] S. Floerchinger, E. Grossi, and J. Lion, Fluid dynamics of heavy ion collisions with mode expansion, Phys. Rev. C 100, 014905 (2019).

[98] M. Dumbser, F. Guercilena, S. Köppel, L. Rezzolla, and O. Zanotti, Conformal and covariant z4 formulation of the Einstein equations: Strongly hyperbolic first-order reduction and solution with discontinuous galerkin schemes, Phys. Rev. D 97, 084053 (2018).

[99] J. S. Hesthaven and T. Warburton, Nodal Discontinuous Galerkin Methods: Algorithms, Analysis, and Applications, 1st ed. (Springer, New York, 2007).

[100] A. Koenigstein, M. J. Steil, J. Braun, M. Buballa, E. Grossi, D. H. Rischke, and N. Wink (to be published).
[101] B. Knorr, Ising and Gross-Neveu model in next-to-leading order, Phys. Rev. B 94, 245102 (2016).

[102] J. Borchardt and B. Knorr, Solving functional flow equations with pseudo-spectral methods, Phys. Rev. D 94, 025027 (2016).

[103] B. Knorr, Critical chiral Heisenberg model with the functional renormalization group, Phys. Rev. B 97, 075129 (2018).

[104] L. Dabelow, H. Gies, and B. Knorr, Momentum dependence of quantum critical Dirac systems, Phys. Rev. D 99, 125019 (2019).

[105] B. Knorr, Exact solutions and residual regulator dependence in functional renormalisation group flows, J. Phys. A 54, 275401 (2021).

[106] G. Eichmann, C. S. Fischer, and C. A. Welzbacher, Baryon effects on the location of QCD's critical end point, Phys. Rev. D 93, 034013 (2016).

[107] T. D. Cohen, Functional Integrals for QCD at Nonzero Chemical Potential and Zero Density, Phys. Rev. Lett. 91, 222001 (2003).

[108] N. Khan, J. M. Pawlowski, F. Rennecke, and M. M. Scherer, The phase diagram of QC2D from functional methods, arXiv:1512.03673.

[109] W.-J. Fu and J. M. Pawlowski, Correlating the skewness and kurtosis of baryon number distributions, Phys. Rev. D 93, 091501 (2016).

[110] O. Bohr, B. J. Schaefer, and J. Wambach, Renormalization group flow equations and the phase transition in $\mathrm{O}(\mathrm{N})$ models, Int. J. Mod. Phys. A 16, 3823 (2001).

[111] D. F. Litim and J. M. Pawlowski, Completeness and consistency of renormalisation group flows, Phys. Rev. D 66, 025030 (2002).

[112] D. F. Litim and J. M. Pawlowski, Wilsonian flows and background fields, Phys. Lett. B 546, 279 (2002).

[113] G. De Polsi, I. Balog, M. Tissier, and N. Wschebor, Precision calculation of critical exponents in the $O(N)$ universality classes with the nonperturbative renormalization group, Phys. Rev. E 101, 042113 (2020).

[114] J. Braun, W.-J. Fu, C. Huang, F. Ihssen, J. Horak, M. Leonhardt, J. M. Pawlowski, M. Pospiech, F. Rennecke, C. Schneider, R. Wen, N. Wink, and S. Yin, Chiral susceptibility in (2+1)-flavor QC, Phys. Rev. D 102, 056010 (2019).

[115] P. Bastian, M. Blatt, A. Dedner, C. Engwer, R. Klöfkorn, S. Kuttanikkad, M. Ohlberger, and O. Sander, The Distributed and Unified Numerics Environment (DUNE), in Proceedings of the 19th Symposium on Simulation Technique in Hannover, September 12-14 (2006).

[116] A. Dedner, B. Flemisch, and R. Klöfkorn, Advances in DUNE (Springer, New York, 2012).

[117] P. Bastian, F. Heimann, and S. Marnach, Generic implementation of finite element methods in the Distributed and Unified Numerics Environment (DUNE), Kybernetika 46, 294 (2010).

[118] M. Blatt and P. Bastian, The iterative solver template library, in Applied Parallel Computing-State of the Art in Scientific Computing, edited by B. Kagström, E. Elmroth, J. Dongarra, and J. Wasniewski (Springer, New York, 2007), pp. 666-675. 
[119] P. Bastian, M. Blatt, A. Dedner, C. Engwer, R. Klöfkorn, M. Ohlberger, and O. Sander, A generic grid interface for parallel and adaptive scientific computing. Part I: Abstract framework, Comput. 82, 103 (2008).

[120] P. Bastian, M. Blatt, A. Dedner, C. Engwer, R. Klöfkorn, R. Kornhuber, M. Ohlberger, and O. Sander, A generic grid interface for parallel and adaptive scientific computing. Part II: Implementation and tests in DUNE, Comput. 82, 121 (2008).

[121] B. Cockburn and C.-W. Shu, The local discontinuous galerkin method for time-dependent convection-diffusion systems, SIAM J. Numer. Anal. 35, 2440 (1998). 